\title{
Transverse Vibration Analysis of Composite Plates with Multiple Distributed Composite Patches
}

\author{
Yan Guo, ${ }^{1}$ Yanan Jiang $\mathbb{D}^{1},{ }^{1}$ Bin Huang $\mathbb{D D}^{2,3}$ and Ji Wang ${ }^{3}$ \\ ${ }^{1}$ College of Science \& Technology, Ningbo University, Ningbo 315300, China \\ ${ }^{2}$ Key Laboratory of Impact and Safety Engineering (Ningbo University), Ministry of Education, Ningbo 315211, China \\ ${ }^{3}$ Piezoelectric Device Laboratory, Faculty of Mechanical Engineering \& Mechanics, Ningbo University, Ningbo 315211, China
}

Correspondence should be addressed to Yanan Jiang; dongguk19@163.com and Bin Huang; huangbin@nbu.edu.cn

Received 27 October 2020; Revised 9 November 2020; Accepted 17 November 2020; Published 29 November 2020

Academic Editor: Giosuè Boscato

Copyright (C) 2020 Yan Guo et al. This is an open access article distributed under the Creative Commons Attribution License, which permits unrestricted use, distribution, and reproduction in any medium, provided the original work is properly cited.

Transverse vibration of rectangular composite plates with multiple distributed composite patches is analyzed in this paper. Because of the geometric discrepancy between the plate and patch, analytical solutions are usually hard to achieve. The present model is formulated by using the Rayleigh-Ritz method and adopting various types of modal shape functions of uniform beam as admissible functions for different boundary conditions. The total system energies are calculated by adding the energies of the substrate plate and the energies of the patches. By imposing the displacement-matching condition at the patch domains, the coordinate systems of the substrate plate and patches are coupled. By means of the present method, it is very convenient and efficient to build the system governing equations and solve the eigenvalue problem. For the composite patches, they are also assumed to be symmetrically layered and have the same layer stacking sequence with the substrate laminate. The effects of layer stacking sequence, modulus ratio, aspect ratio, and boundary conditions on the natural frequencies are investigated and discussed. The results are also compared with the existed benchmark solutions and FEM solutions for validation. The numerical results demonstrate that the proposed approach is computationally very efficient and accurate and can be used as a tool to solve transverse vibration problems of composite plate with multiple composite patches.

\section{Introduction}

Composite patch structures have been widely used in the repair of aircraft, marine ships, and so on, and they are usually used to reinforce structures or repair damaged parts $[1,2]$. By using the patch technology, it can quickly repair and enhance the strength and rigidity of the components, which is beneficial to delay the generation and expansion of cracks in the damaged structures. Since we know it is more effective to connect the patches and the composite substrates by pasting or embedding than the threaded bolt and rivet connection because of the need to open holes that cause stress concentration, composite patch structures play a very important role in many fields. It can not only withstand large loads but also locally strengthen the structures and transfer the strain energy of the stress concentration point.
In recent years, research on layered composite patch structures has received more and more attentions [3, 4]. There are numerous theoretical and experimental works on the application of composite patch structures which demonstrate the engineering significance of their application $[5,6]$. Some scholars have studied the mechanical response of structures after pasting composite patch on the metal substrate through experiments and numerical calculation methods, revealing the complex three-dimensional stress distributions near the free edge of composite patch, where damage such as delamination or crack propagation may be initiated. Ivañez et al. [7] conducted an experimental study of the impact behavior of patch-repaired laminates which are subjected to a broad range of low-velocity impact energies. They made a comparison between intact laminates and double-sided patch repairs in terms of contact load, absorbed energy, and damage area. Mathias and Grediac [8] 
pasted composite patches on the metal substrate of Airbus aircraft, which greatly improved the strength and service life of the metal substrate. Baker [9] used composite patches to repair the cracked aircraft wings, which effectively prevented the cracks from spreading and improved the service life. Schubbe and Mall [10] also found that the use of boron/ epoxy resin composite patches can effectively prevent the propagation of cracks in aluminum plates. Their experimental results showed that the service life of aluminum plates increased four to five times. Cho and Sun [11] studied the thermal stress caused by the use of composite patches for aircraft structural maintenance. They studied the methods of lowering thermal stress through experiment and calculated the thermal residual stress by using a finite element model. Lee et al. $[12,13]$ studied the free-edge stress distributions of composite patch bonded to an isotropic substrate under extension and bending loads. They adopted a simple shear lag model to model the shear stress in the adhesive layer and predicted the three-dimensional stresses based on the stress function-based approach. Through the abovementioned studies, it can be found that the use of patch technology can significantly decrease the stress concentration, increase the strength of substrate with cracks, slow the spread of cracks, and significantly improve the local strength of structures.

With additional patches on substrate composites, it not only affects the structural strength, stress distribution, and failure evolution but also affects the dynamic characteristics of structures [14]. There are many works related to the piezoelectric patches on composite substrates for the analysis of vibration suppression [15], optimal control [16], energy harvesting [17], and structure health monitoring [18]. Huang and Kim [19-21] investigated the transient vibration of composite laminate with surface-bonded piezoelectric actuators and sensors. They found the effect of delamination and debonding of actuator and sensor on the vibration characteristics. Araujo et al. [22] investigated the vibration of laminated soft core sandwich plates with piezoelectric patches by the finite element method. Paknejad et al. [17] provided an analytical solution of piezoelectric patch on multilayer composite beams for energy harvesting. Schulz et al. [23] investigated the optimal piezoelectric patch allocation on composite structures for vibration control based on GA and modal LQR and found the best position of patch around the center of the shell.

However, with regard to composite laminate with single or multiple composite patches, there are few works considering both the effects of patch mass and stiffness on the vibration characteristics. The discrepancy of geometric dimensions results in the difficulty of analytical modeling of patch structures. Thus, the patch structures are usually treated as point mass or distributed mass. Alibeigloo et al. $[24,25]$ investigated the free vibration and forced vibration of antisymmetric laminated plates with distributed patch mass by using the third-order shear deformation theory. They investigated the effects of size and location of the area of the patch, frequency ratio, and mass ratio on the response of the plate. However, they neglected the effect of patch stiffness on the dynamic characteristics although it may be very weak. Vaziri and Nayeb-Hashemi [26] studied the dynamic response of a repaired composite with bonded patch under a harmonic peeling load and found the elastic modulus of the adhesive layer dominates the mechanism of deformation of the repaired beam and its vibration characteristics. In a word, it can be clearly found there is not many works focusing on the modeling of multiple composite laminated patches on composite substrates considering the effects of both mass and stiffness.

In this work, we will investigate the transverse vibration behaviors of composite laminate with surface-bonded patches considering both patch mass and patch stiffness by using the Rayleigh-Ritz method. Although there are a lot of plate and shell theories, such as Reddy's first-order shear deformation theory (FSDT), nonlocal theories, and fourvariable theory, which are very efficient in solving free vibration and nonlinear vibration, dynamic stability and viscoelastic behavior of composite laminates, functionally graded carbon nanotube-reinforced structures (FG-CNT), and porous microplates and skew plates under thermal and mechanical loadings, the Rayleigh-Ritz method is still very simple and efficient in solving free vibration of patched structures [27, 28]. The mathematical model is built with various modal shape functions of uniform beam with different boundary conditions. Because of the geometric discrepancy, the present method uses different coordinate systems for the substrate plate and patches, but they are coupled by applying the displacement-matching conditions $[29,30]$. By using the independent coordinates, it is mathematically more convenient to build the model. We solve the transverse vibration problem with multiple patches on the substrate laminate. The cross-ply and angle-ply laminates with clamped and simply supported boundary conditions are chosen for numerical analysis. The effects of parameters, such as patch number and patch size, aspect ratio, and modulus ratio, on the natural frequencies are also investigated. The present results are compared with those well-known published results and FEM results. The proposed method is very efficient in predicting the transverse vibration of composite laminates with multiple composite patches and can be used as a design tool for modeling of patched composite structures.

\section{Mathematical Modeling}

In this work, we consider a rectangular composite plate with surface-bonded composite patches, as shown in Figure 1. For simplicity, we assume that the patch and composite plate have the same layer stacking sequence and thickness. Their dimensions and the relative location of $k$ th patch are also shown in the figure. The plate has length $a$ in the $x$ direction and width $b$ in the $y$ direction. The dimension of $k$ th patch is $a_{\mathrm{pk}}$ by $b_{\mathrm{pk}}$. Two independent coordinate systems $(x, y, z)$ and $\left(x_{\mathrm{pk}}, y_{\mathrm{pk}}, z_{\mathrm{pk}}\right)$ are used for the composite plate and $k$ th patch, respectively. By defining the nondimensional coordinates for plane axes, we can have the following relation: 


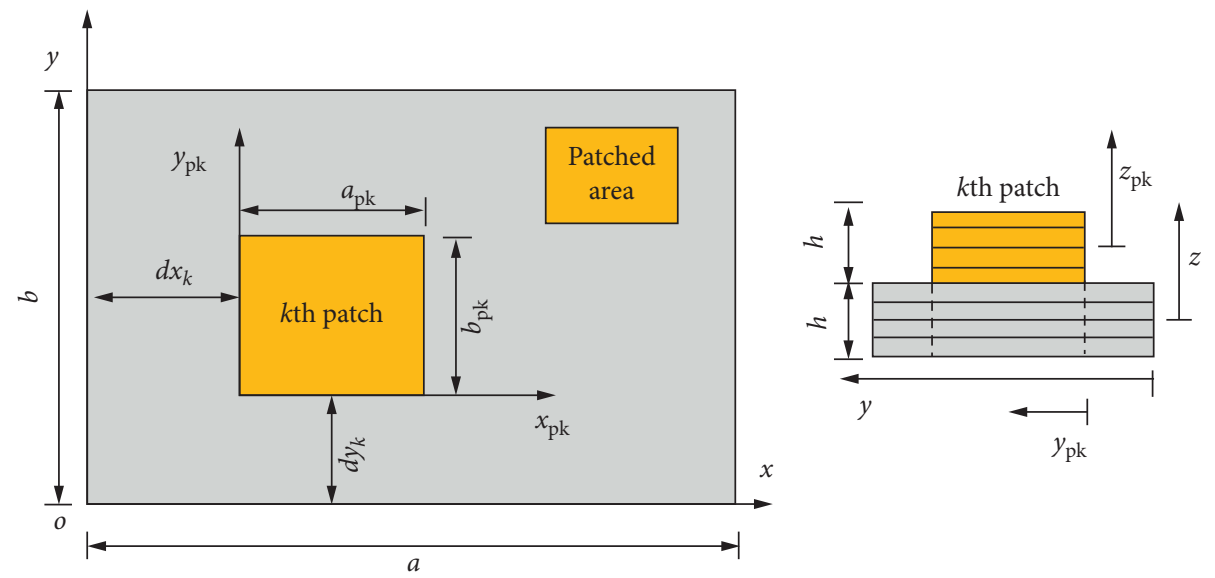

FIGURE 1: Geometry of a composite plate with surface-bonded composite patches and coordinate systems.

$$
\begin{aligned}
\xi & =\frac{x}{a}, \\
\eta & =\frac{y}{b}, \\
\xi_{\mathrm{pk}} & =\frac{x_{\mathrm{pk}}}{a_{\mathrm{pk}}}, \\
\eta_{\mathrm{pk}} & =\frac{y_{\mathrm{pk}}}{b_{\mathrm{pk}}} .
\end{aligned}
$$

We can build the relation between the two coordinates as follows and further use it to couple the two systems:

$$
\begin{aligned}
& \xi=\frac{d_{x k}}{a}+\frac{a_{\mathrm{pk}}}{a} \xi_{\mathrm{pk}}, \\
& \eta=\frac{d_{y k}}{b}+\frac{b_{\mathrm{pk}}}{b} \eta_{\mathrm{pk}} .
\end{aligned}
$$

For a composite laminate with general orthotropic material properties, the constitutive equation for $k$ th laminate can be given by

$$
\left\{\begin{array}{c}
\sigma_{1} \\
\sigma_{2} \\
\sigma_{4} \\
\sigma_{5} \\
\sigma_{6}
\end{array}\right\}^{(k)}=\left[\begin{array}{ccccc}
Q_{11} & Q_{12} & 0 & 0 & Q_{16} \\
Q_{12} & Q_{22} & 0 & 0 & Q_{26} \\
0 & 0 & Q_{44} & Q_{45} & 0 \\
0 & 0 & Q_{45} & Q_{55} & 0 \\
Q_{16} & Q_{26} & 0 & 0 & Q_{66}
\end{array}\right]^{(k)}\left\{\begin{array}{c}
\varepsilon_{1} \\
\varepsilon_{2} \\
\varepsilon_{4} \\
\varepsilon_{5} \\
\varepsilon_{6}
\end{array}\right\}
$$

where $\sigma, \varepsilon$, and $Q$ are the stress, strain, and stiffness matrices, respectively. The superscript $(k)$ represents $k$ th lamina.

For transverse vibration analysis, the kinematic and potential energies can be calculated for the plate and patch separately. And then, the sum of them is the total system kinematic and potential energies. Therefore, they have the following forms for the substrate composite plate and the $k$ th composite patch:

$$
\begin{aligned}
T & =\frac{1}{2} \rho h \int_{0}^{a} \int_{0}^{b} \dot{w}(x, y, t)^{2} \mathrm{~d} x \mathrm{~d} y, \\
V & =\frac{1}{2} \int_{0}^{a} \int_{0}^{b}\left(D_{11}\left(\frac{\partial^{2} w}{\partial x^{2}}\right)^{2}+2 D_{12} \frac{\partial^{2} w}{\partial x^{2}} \frac{\partial^{2} w}{\partial y^{2}}+4 D_{66}\left(\frac{\partial^{2} w}{\partial x \partial y}\right)^{2}+D_{22}\left(\frac{\partial^{2} w}{\partial y^{2}}\right)^{2}\right) \mathrm{d} x \mathrm{~d} y, \\
T_{\mathrm{pk}} & =\frac{1}{2} \rho h \int_{0}^{a_{\mathrm{pk}}} \int_{0}^{b_{\mathrm{pk}}} \dot{w}_{\mathrm{pk}}\left(x_{\mathrm{pk}}, y_{\mathrm{pk}}, t\right)^{2} \mathrm{~d} x_{\mathrm{pk}} \mathrm{d} y_{\mathrm{pk}}, \\
V_{\mathrm{pk}} & =\frac{1}{2} \int_{0}^{a_{\mathrm{pk}}} \int_{0}^{b_{\mathrm{pk}}}\left(D_{11}\left(\frac{\partial^{2} w_{\mathrm{pk}}}{\partial x_{\mathrm{pk}}^{2}}\right)^{2}+2 D_{12} \frac{\partial^{2} w_{\mathrm{pk}}}{\partial x_{\mathrm{pk}}^{2}} \frac{\partial^{2} w_{\mathrm{pk}}}{\partial y_{\mathrm{pk}}^{2}}+4 D_{66}\left(\frac{\partial^{2} w_{\mathrm{pk}}}{\partial x_{\mathrm{pk}} \partial y_{\mathrm{pk}}}\right)^{2}+D_{22}\left(\frac{\partial^{2} w_{\mathrm{pk}}}{\partial y_{\mathrm{pk}}^{2}}\right)^{2}\right) \mathrm{d} x_{\mathrm{pk}} \mathrm{d} y_{\mathrm{pk}},
\end{aligned}
$$


where $w(x, y, t)$ and $w_{\mathrm{pk}}(x, y, t)$ represent the deflections of the composite plate and $k$ th patch. $\rho$ is the material density and $D_{i j}$ is the bending stiffness which can be calculated by the following equation:

$$
\begin{aligned}
D_{i j} & =\int_{-h / 2}^{h / 2} Q_{i j} z^{2} \mathrm{~d} z \\
& =\sum_{k=1}^{N} \int_{z_{k}}^{z_{k+1}} Q_{i j}^{(k)} z^{2} \mathrm{~d} z=\frac{1}{3} \sum_{k=1}^{N} Q_{i j}^{(k)}\left(z_{k+1}^{3}-z_{k}^{3}\right) .
\end{aligned}
$$

The assumed mode method assumes a solution of the boundary-value problem and uses it in conjunction with Lagrange's equations to obtain an approximate formulation of the equations of motion. The solution is assumed as

$$
\begin{aligned}
w(\xi, \eta, t) & =\Phi(\xi, \eta) q(t), \\
w_{\mathrm{pk}}\left(\xi_{\mathrm{pk}}, \eta_{\mathrm{pk}}, t\right) & =\Phi_{\mathrm{pk}}\left(\xi_{\mathrm{pk}}, \eta_{\mathrm{pk}}\right) q_{\mathrm{pk}}(t),
\end{aligned}
$$

where the admissible functions $\Phi$ and $\Phi_{\mathrm{pk}}$ and the generalized coordinates $q(t)$ and $q_{\mathrm{pk}}(t)$ consist of $n$ and $n_{\mathrm{pk}}$ terms and have the following matrix forms for the plate and $k$ th patch:

$$
\begin{aligned}
\Phi(\xi, \eta) & =\left[\begin{array}{llll}
\Phi_{1} & \Phi_{2} & \ldots & \Phi_{n}
\end{array}\right]_{1 \times n}, \\
q(t) & =\left[\begin{array}{llll}
q_{1} & q_{2} & \ldots & q_{n}
\end{array}\right]_{n \times 1}^{T}, \\
\Phi_{p k}\left(\xi_{p k}, \eta_{p k}\right) & =\left[\begin{array}{llll}
\Phi_{p k 1} & \Phi_{p k 2} & \ldots & \Phi_{p k n_{p k}}
\end{array}\right]_{1 \times n_{p k}}, \\
q_{p k}(t) & =\left[\begin{array}{llll}
q_{p k 1} & q_{p k 2} & \ldots & q_{p k n_{p k}}
\end{array}\right]_{p p k}^{T} .
\end{aligned}
$$

By using the nondimensional coordinates, equation (1), and substituting it into equation (4), the kinematic and potential energy functions can be expressed by the matrix form for the plate,

$$
\begin{aligned}
& T=\frac{1}{2} \dot{q}^{T} M \dot{q}, \\
& V=\frac{b}{2 a^{3}} q^{T} \bar{K} q,
\end{aligned}
$$

where

$$
\begin{aligned}
& M=\rho h a b \bar{M}, \\
& K=\frac{b}{a^{3}} \bar{K} \\
& \bar{M}=\int_{0}^{1} \int_{0}^{1} \Phi^{T} \Phi \mathrm{d} \xi \mathrm{d} \eta, \\
& \bar{K}=\int_{0}^{1} \int_{0}^{1}\left(D_{11} \frac{\partial^{2} \Phi^{T}}{\partial \xi^{2}} \frac{\partial^{2} \Phi}{\partial \xi^{2}}+2 D_{12} \alpha^{2} \frac{\partial^{2} \Phi^{T}}{\partial \xi^{2}} \frac{\partial^{2} \Phi}{\partial \eta^{2}}+4 D_{66} \alpha^{2} \frac{\partial^{2} \Phi^{T}}{\partial \xi \partial \eta} \frac{\partial^{2} \Phi}{\partial \xi \partial \eta}+D_{22} \alpha^{4} \frac{\partial^{2} \Phi^{T}}{\partial \eta^{2}} \frac{\partial^{2} \Phi}{\partial \eta^{2}}\right) \mathrm{d} \xi \mathrm{d} \eta,
\end{aligned}
$$

and for the $k$ th patch,

where

$$
\begin{aligned}
& T_{\mathrm{pk}}=\frac{1}{2} \dot{q}_{\mathrm{pk}}^{T} M_{\mathrm{pk}} \dot{q}_{\mathrm{pk}}, \\
& V_{\mathrm{pk}}=\frac{1}{2} q_{\mathrm{pk}}^{T} K_{\mathrm{pk}} q_{\mathrm{pk}},
\end{aligned}
$$

$$
\begin{aligned}
M_{\mathrm{pk}} & =\rho h a_{\mathrm{pk}} b_{\mathrm{pk}} \bar{M}_{\mathrm{pk}}, \\
K_{\mathrm{pk}} & =\frac{b_{\mathrm{pk}}}{a_{\mathrm{pk}}^{3}} \overline{\mathrm{pk}}_{\mathrm{pk}}, \\
\bar{M}_{\mathrm{pk}} & =\int_{0}^{1} \int_{0}^{1} \Phi_{\mathrm{pk}}^{T} \Phi_{\mathrm{pk}} \mathrm{d} \xi_{\mathrm{pk}} \mathrm{d} \eta_{\mathrm{pk}},
\end{aligned}
$$




$$
\bar{K}_{\mathrm{pk}}=\int_{0}^{1} \int_{0}^{1}\left(\begin{array}{c}
D_{11} \frac{\partial^{2} \Phi_{\mathrm{pk}}^{T}}{\partial \xi_{\mathrm{pk}}^{2}} \frac{\partial^{2} \Phi_{\mathrm{pk}}}{\partial \xi_{\mathrm{pk}}^{2}}+2 D_{12} \alpha_{\mathrm{pk}}^{2} \frac{\partial^{2} \Phi_{\mathrm{pk}}^{T}}{\partial \xi_{\mathrm{pk}}^{2}} \frac{\partial^{2} \Phi_{\mathrm{pk}}}{\partial \eta_{\mathrm{pk}}^{2}} \\
+4 D_{66} \alpha_{p k}^{2} \frac{\partial^{2} \Phi_{\mathrm{pk}}^{T}}{\partial \xi_{\mathrm{pk}} \partial \eta_{\mathrm{pk}}} \frac{\partial^{2} \Phi_{\mathrm{pk}}}{\partial \xi_{\mathrm{pk}} \partial \eta_{\mathrm{pk}}}+D_{22} \alpha_{\mathrm{pk}}^{4} \frac{\partial^{2} \Phi_{\mathrm{pk}}^{T}}{\partial \eta_{\mathrm{pk}}^{2}} \frac{\partial^{2} \Phi_{\mathrm{pk}}}{\partial \eta_{\mathrm{pk}}^{2}}
\end{array}\right) \mathrm{d} \xi_{\mathrm{pk}} \mathrm{d} \eta_{\mathrm{pk}}
$$

The length-to-width ratios $\alpha$ and $\alpha_{p k}$ are defined by $\alpha=a / b$ and $\alpha_{\mathrm{pk}}=a_{\mathrm{pk}} / b_{\mathrm{pk}}$ in the above equations. The admissible functions are further separated into the admissible functions in each direction as follows:

$$
\begin{gathered}
\Phi_{i}(\xi, \eta)=\phi_{i}(\xi) \psi_{i}(\eta), \quad i=1,2, \ldots, n, \\
\Phi_{p k i}\left(\xi_{p k}, \eta_{p k}\right)=\phi_{p k i}\left(\xi_{p k}\right) \psi_{p k i}\left(\eta_{p k}\right), \quad i=1,2, \ldots, n_{p k} .
\end{gathered}
$$

Substituting the admissible function, equation (10a), into the nondimensionalized mass and stiffness matrices of the plate, equations (9e) and (9f), these matrices can be expressed in terms of the following integrations:

$$
\begin{aligned}
\bar{M}_{i j}= & X 1_{i j} Y 1_{i j}, \quad(i, j=1,2, \ldots, m), \\
\bar{K}_{i j}= & D_{11} X 3_{i j} Y 1_{i j}+2 D_{12} \alpha^{2} X 4_{i j} Y 4_{i j} \\
& +4 D_{66} \alpha^{2} X 2_{i j} Y 2_{i j}+D_{22} \alpha^{4} X 1_{i j} Y 3_{i j},
\end{aligned}
$$

where

$$
\begin{aligned}
& X 1_{i j}=\int_{0}^{1} \phi_{i} \phi_{j} \mathrm{~d} \xi, \\
& X 2_{i j}=\int_{0}^{1} \phi_{i}^{\prime} \phi_{j}^{\prime} \mathrm{d} \xi, \\
& X 3_{i j}=\int_{0}^{1} \phi_{i}^{\prime \prime} \phi_{j}^{\prime \prime} \mathrm{d} \xi, \\
& X 4_{i j}=\int_{0}^{1} \phi_{i} \phi_{j}^{\prime \prime} \mathrm{d} \xi, \\
& Y 1_{i j}=\int_{0}^{1} \psi_{i} \psi_{j} \mathrm{~d} \eta, \\
& Y 2_{i j}=\int_{0}^{1} \psi_{i}^{\prime} \psi_{j}^{\prime} \mathrm{d} \eta, \\
& Y 3_{i j}=\int_{0}^{1} \psi_{i}^{\prime \prime} \psi_{j}^{\prime \prime} \mathrm{d} \eta, \\
& Y 4_{i j}=\int_{0}^{1} \psi_{i} \psi_{j}^{\prime \prime} \mathrm{d} \eta, \quad(i, j=1,2, \ldots, n),
\end{aligned}
$$

and the superscripts (') and (") represent the first and second derivatives, respectively.

If we choose $N$ admissible functions $\chi_{i}(i=1,2, \ldots, N)$ and $\gamma_{i}(i=1,2, \ldots, N)$ as follows in $x$ and $y$ directions, respectively, the total combination of admissible functions equals $N^{2}$.

$$
\begin{gathered}
\phi_{k}= \begin{cases}\chi_{1}, & 1 \leq k \leq N, \\
\chi_{2}, & N+1 \leq k \leq 2 N, \\
\chi_{3}, & 2 N+1 \leq k \leq 3 N, \\
& \vdots \\
\chi_{N}, & (N-1) N+1 \leq k \leq N^{2},\end{cases} \\
\psi_{k}= \begin{cases}\gamma_{k}, & 1 \leq k \leq N, \\
\gamma_{k-N}, & N+1 \leq k \leq 2 N, \\
\gamma_{k-2 N}, & 2 N+1 \leq k \leq 3 N, \\
\gamma_{k-(N-1) N}, & (N-1) N+1 \leq k \leq N^{2} .\end{cases}
\end{gathered}
$$

Substituting equation (12) into equations (11c) and (11d), the coefficients can be calculated by the following integrations:

$$
\begin{aligned}
& \Sigma_{i j}=\int_{0}^{1} \chi_{i} \chi_{j} \mathrm{~d} \xi, \\
& \bar{\Sigma}_{i j}=\int_{0}^{1} \chi_{i}^{\prime} \chi_{j}^{\prime} \mathrm{d} \xi, \\
& \widehat{\Sigma}_{i j}=\int_{0}^{1} \chi_{i}^{\prime \prime} \chi_{j}^{\prime \prime} \mathrm{d} \xi, \\
& \widetilde{\Sigma}_{i j}=\int_{0}^{1} \chi_{i} \chi_{j}^{\prime \prime} \mathrm{d} \xi, \\
& \bar{\Gamma}_{i j}=\int_{0}^{1} \gamma_{i} \gamma_{j} \mathrm{~d} \eta, \\
& \widehat{\Gamma}_{i j}=\int_{0}^{1} \gamma_{i}^{\prime} \gamma_{j}^{\prime} \mathrm{d} \eta, \\
& \widetilde{\Gamma}_{i j}=\int_{0}^{1} \gamma_{i} \gamma_{j}^{\prime \prime} \mathrm{d} \eta, \quad(13 \mathrm{~b})
\end{aligned}
$$

Therefore, the matrices $(X 1, X 2, X 3, X 4)$ and $(Y 1, Y 2, Y 3$, $Y 4)$ can be approximated with proper assumption of admissible functions. The next step is to choose proper admissible functions for plates with different boundary conditions. We firstly choose three types of modal shape functions of uniform beam as admissible functions in the $x$ 
direction. The details of modal shape functions are given as follows:

(i) Simply supported boundary condition:

$$
\chi_{i}=\sqrt{2} \sin (i \pi \xi), \quad i=1,2, \ldots, N .
$$

(ii) Clamped boundary condition:

$$
\begin{gathered}
\chi_{i}=\cosh \left(\lambda_{i} \xi\right)-\cos \left(\lambda_{i} \xi\right)-\sigma_{i}\left(\sinh \left(\lambda_{i} \xi\right)-\sin \left(\lambda_{i} \xi\right)\right) \\
i=1,2, \ldots, N
\end{gathered}
$$

where $\lambda_{i}=4.730,7.853,10.996,14.137, \ldots$, and $\sigma_{i}=\left(\cosh \left(\lambda_{i}\right)-\cos \left(\lambda_{i}\right)\right) /\left(\sinh \left(\lambda_{i}\right)-\sin \left(\lambda_{i}\right)\right)$.

(iii) Free-free boundary condition:

$$
\begin{gathered}
\chi_{1}=1, \\
\chi_{2}=\sqrt{12}(\xi-1), \\
\chi_{i+2}=\cosh \left(\lambda_{i} \xi\right)+\cos \left(\lambda_{i} \xi\right)-\sigma_{i}\left(\sinh \left(\lambda_{i} \xi\right)+\sin \left(\lambda_{i} \xi\right)\right), \\
i=1,2, \ldots, N-2 .
\end{gathered}
$$

Thus, substituting equations (14)-(16) into the integration equation (13), we can obtain the coefficient matrices $(X 1, X 2, X 3, X 4)$. Similarly, we can also calculate the coefficient matrices $(Y 1, Y 2, Y 3, Y 4)$ by assuming proper admissible function and performing the integrations in the $y$ direction. After solving these matrices, we can obtain the nondimensionalized mass matrix $\bar{M}$ and stiffness matrix $\bar{K}$ from equations (11a) and (11b) for different boundary conditions. By the same method, we can obtain the nondimensionalized mass and stiffness matrices for the patch domain, but only with the free-free boundary condition. For the sake of brevity, it will not be introduced again.

The displacement-matching condition has to be applied for all the patches. For the $k$ th patch, it must satisfy the following relation:

$$
w_{\mathrm{pk}}\left(\xi_{\mathrm{pk}}, \eta_{\mathrm{pk}}, t\right)=w(\xi, \eta, t) .
$$

By means of equations (6) and (10), the above equation becomes

$$
\begin{aligned}
& \sum_{j=1}^{n_{p k}} \Phi_{p k j}\left(\xi_{\mathrm{pk}}, \eta_{\mathrm{pk}}\right) q_{p k j}(t)=\sum_{j=1}^{n_{p k}} \phi_{p k j}\left(\xi_{\mathrm{pk}}\right) \psi_{p k j}\left(\eta_{\mathrm{pk}}\right) q_{p k j}(t) \\
& =\sum_{k=1}^{n} \Phi_{k}(\xi, \eta) q_{k}(t)=\sum_{k=1}^{n} \phi_{k}(\xi) \psi_{k}(\eta) q_{k}(t)
\end{aligned}
$$

Multiplying $\phi_{p k i}\left(\xi_{\mathrm{pk}}\right) \psi_{p k i}\left(\eta_{\mathrm{pk}}\right)$ and performing integration to equation (18), we obtain

$$
\begin{aligned}
& \sum_{j=1}^{n_{p k}} \int_{0}^{1} \int_{0}^{1} \phi_{p k i}\left(\xi_{\mathrm{pk}}\right) \psi_{p k i}\left(\eta_{\mathrm{pk}}\right) \phi_{p k j}\left(\xi_{\mathrm{pk}}\right) \psi_{p k j}\left(\eta_{\mathrm{pk}}\right) \mathrm{d} \xi_{\mathrm{pk}} \mathrm{d} \eta_{\mathrm{pk}} q_{p k j}(t) \\
& =\sum_{k=1}^{n} \int_{0}^{1} \int_{0}^{1} \phi_{p k i}\left(\xi_{\mathrm{pk}}\right) \psi_{p k i}\left(\eta_{\mathrm{pk}}\right) \phi_{k}(\xi) \psi_{k}(\eta) \mathrm{d} \xi_{\mathrm{pk}} \mathrm{d} \eta_{\mathrm{pk}} q_{k}(t), \quad\left(i=1,2, \ldots, n_{\mathrm{pk}}\right) .
\end{aligned}
$$

We can simply equation (19) by using the orthogonal property of eigenfunctions of the uniform beam and obtain the following equation:

$$
\begin{aligned}
q_{p k i}(t) & =\sum_{k=1}^{n} \int_{0}^{1} \phi_{p k i}\left(\xi_{\mathrm{pk}}\right) \phi_{k}(\xi) \mathrm{d} \xi_{\mathrm{pk}} \int_{0}^{1} \psi_{p k i}\left(\eta_{\mathrm{pk}}\right) \psi_{k}(\eta) \mathrm{d} \eta_{\mathrm{pk}} q_{k}(t) \\
& =\sum_{k=1}^{n}\left(S_{\mathrm{pk}}\right)_{i k} q_{k}(t), \quad\left(i=1,2, \ldots, n_{\mathrm{pk}}\right) .
\end{aligned}
$$

Thus, the generalized coordinate transformation equation for the $k$ th patch can be expressed as follows:

$$
q_{\mathrm{pk}}=S_{\mathrm{pk}} q
$$

where $S_{\mathrm{pk}}$ is $n_{\mathrm{pk}}$ by $n$ transformation matrix describing the relation between the global coordinate and the coordinate of the $k$ th patch that can be calculated with the help of equation (2).

For the plate with $N_{p}$ patches, the total kinetic and potential energies of the system can be obtained by the summation of individual energy as follows: 


$$
\begin{aligned}
T_{\text {total }}= & \frac{1}{2} \dot{q}^{T} M \dot{q}+\sum_{k=1}^{N_{p}} \frac{1}{2} \dot{q}_{\mathrm{pk}}^{T} M_{\mathrm{pk}} \dot{q}_{\mathrm{pk}}=\frac{1}{2} \dot{q}^{T} M \dot{q} \\
& +\sum_{k=1}^{N_{p}} \frac{1}{2} \dot{q}^{T} S_{\mathrm{pk}}^{T} M_{\mathrm{pk}} S_{\mathrm{pk}} \dot{q}=\frac{1}{2} \dot{q}^{T} M_{T} \dot{q}, \\
V_{\text {total }}= & \frac{1}{2} q^{T} K q+\sum_{k=1}^{N_{p}} \frac{1}{2} q_{\mathrm{pk}}^{T} K_{\mathrm{pk}} q_{\mathrm{pk}}=\frac{1}{2} q^{T} K q \\
& +\sum_{k=1}^{N_{p}} \frac{1}{2} q^{T} S_{\mathrm{pk}}^{T} K_{\mathrm{pk}} S_{\mathrm{pk}} q=\frac{1}{2} q^{T} K_{T} q,
\end{aligned}
$$

where

$$
\begin{gathered}
M_{T}=M+\sum_{k=1}^{N_{p}} S_{\mathrm{pk}}^{T} M_{\mathrm{pk}} S_{\mathrm{pk}}, \\
K_{T}=K+\sum_{k=1}^{N_{p}} S_{\mathrm{pk}}^{T} K_{\mathrm{pk}} S_{\mathrm{pk}} .
\end{gathered}
$$

Using the nondimensional variables, equation (23) can be rewritten as

$$
\begin{aligned}
M_{T} & =\rho h a b \bar{M}_{T}, \\
K_{T} & =\frac{b}{a^{3}} \bar{K}_{T},
\end{aligned}
$$

where

$$
\begin{gathered}
\bar{M}_{T}=\bar{M}+\sum_{k=1}^{N_{p}}\left(\bar{a}_{\mathrm{pk}} \bar{b}_{\mathrm{pk}}\right) S_{\mathrm{pk}}^{T} \bar{M}_{\mathrm{pk}} S_{\mathrm{pk}}, \\
\bar{K}_{T}=\bar{K}+\sum_{k=1}^{N_{p}} \frac{\bar{b}_{\mathrm{pk}}}{\bar{a}_{\mathrm{pk}}^{3}} S_{\mathrm{pk}}^{T} \bar{K}_{\mathrm{pk}} S_{\mathrm{pk}}, \\
\bar{a}_{\mathrm{pk}}=\frac{a_{\mathrm{pk}}}{a}, \\
\bar{b}_{\mathrm{pk}}=\frac{b_{\mathrm{pk}}}{b} .
\end{gathered}
$$

By substituting the total energy equations into Lagrange's equation, we can obtain the following eigenvalue problem:

$$
\left[K_{T}-\omega^{2} M_{T}\right] A=0,
$$

where $\omega$ is the natural frequency, and the above eigenvalue problem can be solved firstly by using the nondimensionalized mass and stiffness matrices as follows:

$$
\left[\bar{K}_{T}-\varpi^{2} \bar{M}_{T}\right] A=0,
$$

where $₫=\omega \sqrt{\rho h a^{4}}$ is the nondimensionalized natural frequency. Therefore, the eigenvalue problem in equation (26) can be solved.

\section{Numerical Results}

To investigate the numerical results calculated by the present approach, the following material properties and geometric relations are used for the calculations [31]:

$$
\begin{aligned}
& \frac{E_{1}}{E_{2}}=10 \text { or } 20, \\
& \frac{E_{2}}{G_{12}}=2, \\
& \frac{E_{2}}{G_{23}}=5, \\
& G_{12}=G_{13}, \\
& V_{12}=V_{13}=0.25, \\
& \frac{a}{b}=1 \text { or } 2, \\
& \frac{h}{b}=0.1, \\
& \frac{a_{\mathrm{pk}}}{a}=0.1,0.2 \text { or } 0.3 .
\end{aligned}
$$

The accuracy of the present approach for transverse vibration analysis of composite substrate with distributed multiple composite patches is validated by the finite element method and other existed benchmark solutions. For the FEM solutions, they are obtained by using the commercial package ANSYS with shell elements. Three cases of patch numberings and locations are shown in Figure 2, where CASE I represents single patch and CASE II and CASE III represent two and four patches, respectively. We will examine the examples with different layer stacking sequences, patch numbers, patch sizes, modulus ratios, aspect ratios, and boundary conditions in the following sections.

3.1. Cross-Ply Plates ([0/90]s) with Single and Multiple Patches. We start by considering a cross-ply ([0/90]s) plate with single and multiple composite patches and four-edge clamped (CCCC) boundary condition. We firstly present the first nine nondimensional frequencies of three cases with $E 1 /$ $E 2=10$ and $a=b=10 \mathrm{~h}$ and various patch sizes for the present approach in Table 1. It should be noted that the natural frequencies obtained in this work are all nondimensionalized by $\Omega=\omega\left(b^{2} \pi^{2}\right) \sqrt{\rho h / D_{22}}$. From the table, it is found that, for the CCCC boundary condition, the plate has smaller frequencies when it has single or multiple patches compared with the plate without a patch. Moreover, when increasing the patch size, it is found that the 

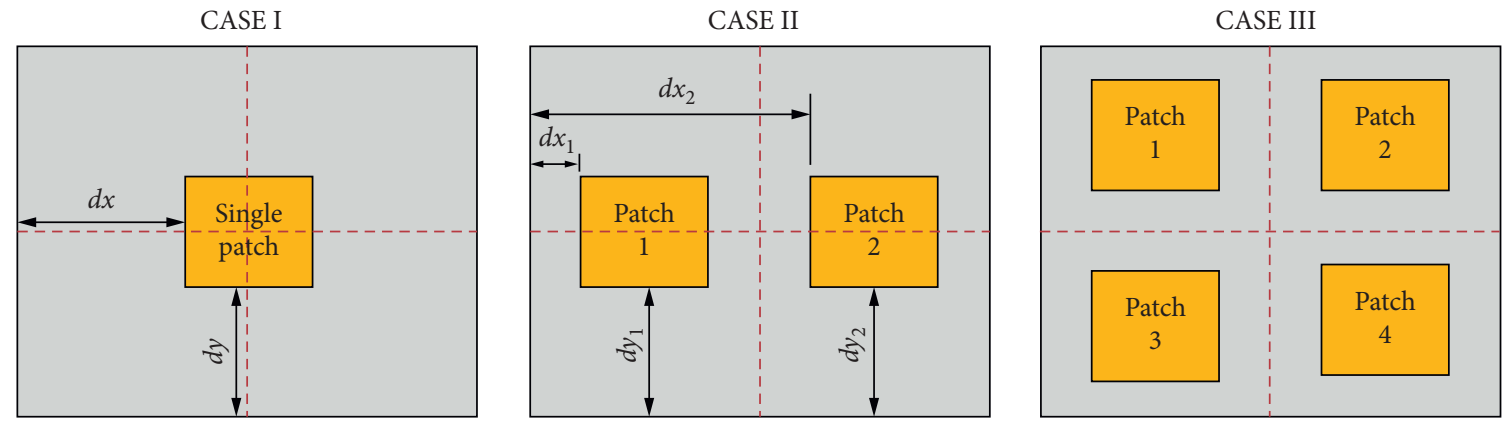

FIgURe 2: Patch numbering and locations on the substrate composite plate.

TABLE 1: Nondimensional frequencies of the cross-ply laminate ([0/90]s) with CCCC boundary condition, $E 1 / E 2=10$, and $a=b=10 \mathrm{~h}$ and for different patch sizes and numbers.

\begin{tabular}{|c|c|c|c|c|c|c|c|c|c|c|c|}
\hline Modes & & & $1 \mathrm{st}$ & 2nd & $3 \mathrm{rd}$ & 4th & 5 th & 6th & 7 th & 8th & 9th \\
\hline \multirow{8}{*}{ CASE I } & \multirow{2}{*}{$0 \times 0$} & Present & 5.288 & 8.178 & 13.220 & 13.618 & 15.079 & 19.171 & 21.347 & 25.409 & 25.831 \\
\hline & & FEM & 5.337 & 8.013 & 12.693 & 13.171 & 14.390 & I & I & I & / \\
\hline & \multirow{2}{*}{$0.1 \times 0.1$} & Present & 5.226 & 8.173 & 13.214 & 13.504 & 15.097 & 19.164 & 21.318 & 25.199 & 25.861 \\
\hline & & FEM & 5.205 & 8.064 & 12.416 & 13.119 & 14.506 & I & 1 & 1 & 1 \\
\hline & \multirow{2}{*}{$0.2 \times 0.2$} & Present & 5.067 & 8.121 & 13.131 & 13.322 & 15.124 & 19.096 & 21.018 & 24.865 & 25.851 \\
\hline & & FEM & 4.942 & 8.284 & 12.243 & 13.102 & 14.708 & l & I & I & I \\
\hline & \multirow{2}{*}{$0.3 \times 0.3$} & Present & 4.878 & 7.951 & 12.860 & 13.301 & 15.046 & 18.997 & 20.504 & 24.853 & 25.556 \\
\hline & & FEM & 4.650 & 8.476 & 12.299 & 13.021 & 14.242 & 1 & 1 & I & 1 \\
\hline \multirow{6}{*}{ CASE II } & \multirow{2}{*}{$0.1 \times 0.1$} & Present & 5.180 & 8.170 & 13.209 & 13.423 & 15.110 & 19.158 & 21.296 & 25.039 & 25.883 \\
\hline & & FEM & 5.085 & 7.788 & 11.798 & 12.895 & 15.463 & I & 1 & 1 & I \\
\hline & \multirow{2}{*}{$0.2 \times 0.2$} & Present & 4.916 & 8.079 & 13.063 & 13.137 & 15.157 & 19.041 & 20.772 & 24.518 & 25.865 \\
\hline & & FEM & 4.928 & 7.174 & 11.992 & 12.559 & 15.160 & 1 & 1 & 1 & I \\
\hline & \multirow[b]{2}{*}{$0.3 \times 0.3$} & Present & 4.624 & 7.790 & 12.599 & 13.131 & 15.022 & 18.886 & 19.958 & 24.565 & 25.355 \\
\hline & & FEM & 4.858 & 6.849 & 11.895 & 12.117 & 13.678 & 1 & I & 1 & 1 \\
\hline \multirow{6}{*}{ CASE III } & \multirow{2}{*}{$0.1 \times 0.1$} & Present & 5.106 & 8.165 & 13.201 & 13.294 & 15.131 & 19.150 & 21.260 & 24.772 & 25.918 \\
\hline & & FEM & 4.998 & 7.767 & 11.749 & 12.708 & 15.103 & I & I & 1 & 1 \\
\hline & \multirow{2}{*}{$0.2 \times 0.2$} & Present & 4.690 & 8.009 & 12.883 & 12.951 & 15.208 & 18.954 & 20.370 & 24.051 & 25.889 \\
\hline & & FEM & 4.801 & 7.087 & 11.955 & 12.221 & 14.971 & I & I & I & I \\
\hline & \multirow{2}{*}{$0.3 \times 0.3$} & Present & 4.274 & 7.536 & 12.185 & 12.925 & 14.982 & 18.730 & 19.201 & 24.230 & 25.030 \\
\hline & & FEM & 4.650 & 6.799 & 11.956 & 12.409 & 14.217 & 1 & I & I & I \\
\hline
\end{tabular}

nondimensional frequencies decrease due to the mass effect. It is also found that the nondimensional frequencies decrease when increasing the patch numbers. With four patches in CASE III, the frequencies are apparently smaller than those of CASE I and CASE II. The first five nondimensional frequencies calculated by the FEM are also provided in Table 1 for comparison. Since we used the shell element in the FEM modeling which has six degrees of freedom (DOF) for each node, it has more frequency values so that we have to pick out the corresponding frequencies by comparing the modal shapes. The FEM results are convergent and obtained with very fine mesh size. We totally used ten thousand elements and fixed boundary condition at the edges. The FEM results show that two methods have tolerable margin of errors and they can be used to efficiently validate the results provided by the present approach. The FEM results also demonstrate the same tendency of frequencies regarding to the patch number and patch size. Thus, the correctness and accuracy of the present approach for CCCC boundary condition are guaranteed. The modal shapes of cross-ply laminate with single patch and patch size $0.3 \times 0.3$ are shown in Figure 3, from the FEM results.

We next investigate the effects of modulus ratio and aspect ratio on the nondimensional frequencies of cross-ply laminate with CCCC boundary condition, as given in $\mathrm{Ta}-$ bles 2 and 3, respectively. When the modulus ratio E1/ $E 2=20$, the natural frequencies increase compared with those of $E 1 / E 2=10$. When the aspect ratio $a=2 \mathrm{~b}$, the nondimensional frequencies significantly decrease compared with those in Table 1 and the fundamental frequency decreases to 2.612 for the plate without a patch. The effects of patch number and patch size can be observed in Tables 2 and 3 , and similar conclusions can be achieved as discussed before.

Four-edge simply supported (SSSS) boundary conditions are also investigated for cross-ply laminates with single and multiple composite patches. We also examine the effects of patch number, patch size, modulus ratio, and aspect ratio on the natural frequencies, and the results are given in Tables 4-6. Table 4 gives the results of SSSS plate with E1/ 


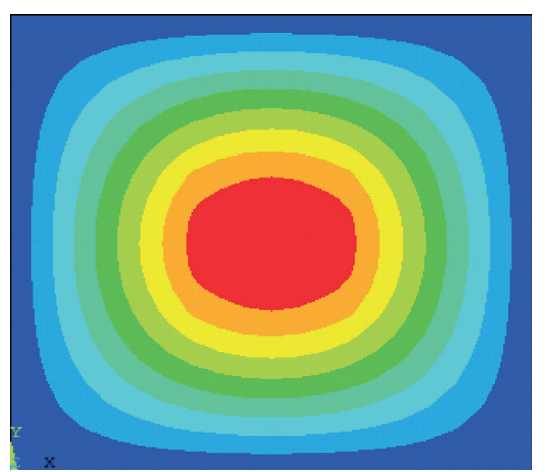

(a)

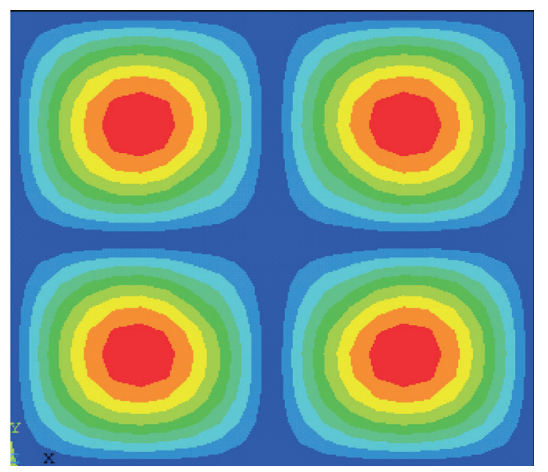

(d)

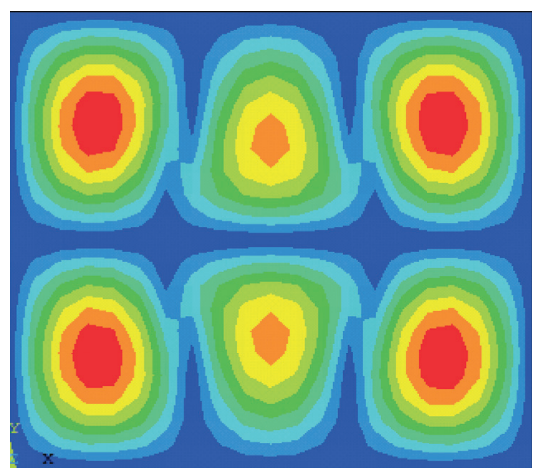

(g)

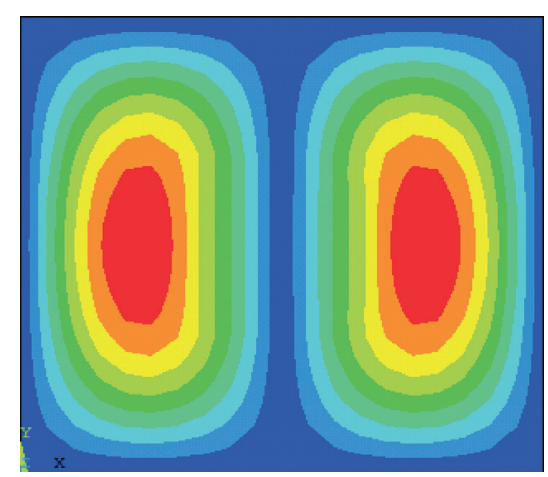

(b)

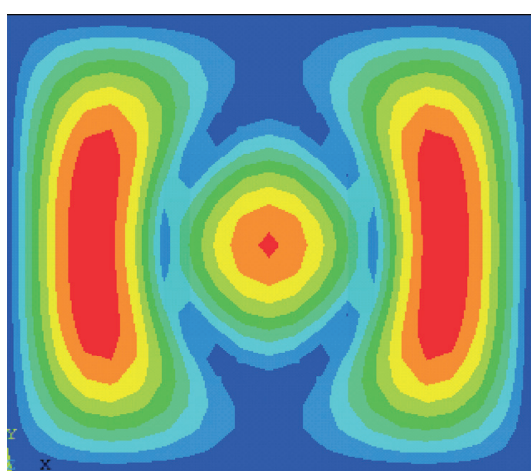

(e)

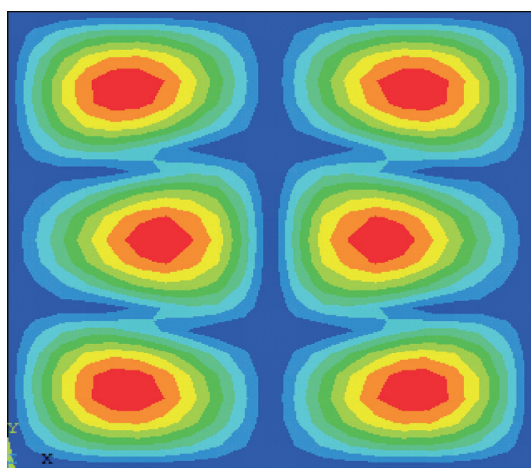

(h)

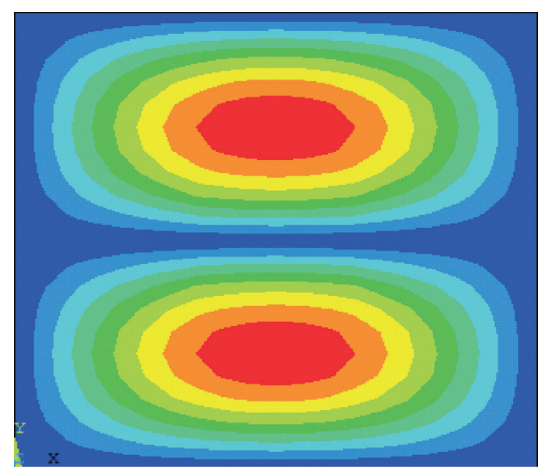

(c)

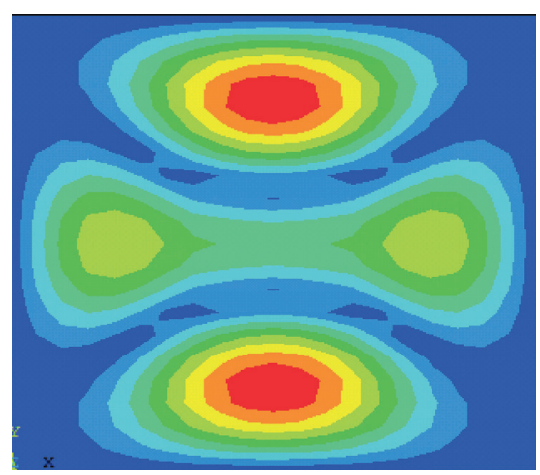

(f)

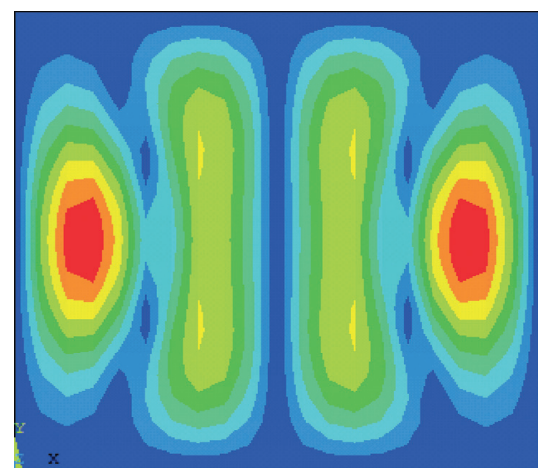

(i)

FIGURe 3: Modal shapes of the cross-ply laminate ([90/-90]s) with single patch (CASE (I) $0.3 \times 0.3)$ on the composite substrate and CCCC boundary condition by the FEM.

TABLE 2: Nondimensional frequencies of the cross-ply laminate ([0/90]s) with CCCC boundary condition, $E 1 / E 2=20$, and $a=b=10 \mathrm{~h}$ and for different patch sizes and numbers.

\begin{tabular}{lcccccccccc}
\hline Modes & & 1st & 2nd & 3rd & 4th & 5th & 6th & 7th & 8th & 9th \\
\hline Present & $0 \times 0$ & 5.711 & 8.350 & 13.622 & 14.602 & 16.094 & 19.709 & 21.263 & 25.955 & 28.241 \\
\hline \multirow{2}{*}{ CASE I } & $0.1 \times 0.1$ & 5.643 & 8.346 & 13.508 & 14.595 & 16.105 & 19.702 & 21.234 & 25.974 & 27.990 \\
& $0.2 \times 0.2$ & 5.471 & 8.292 & 13.327 & 14.502 & 16.114 & 19.633 & 20.932 & 25.944 & 27.581 \\
& $0.3 \times 0.3$ & 5.266 & 8.117 & 13.308 & 14.199 & 16.007 & 19.537 & 20.423 & 25.642 & 27.599 \\
\hline \multirow{3}{*}{ CASE II } & $0.1 \times 0.1$ & 5.593 & 8.343 & 13.426 & 14.590 & 16.113 & 19.697 & 21.212 & 25.988 & 27.781 \\
& $0.2 \times 0.2$ & 5.306 & 8.248 & 13.145 & 14.427 & 16.129 & 19.578 & 20.687 & 25.935 & 27.130 \\
& $0.3 \times 0.3$ & 4.989 & 7.950 & 13.142 & 13.905 & 15.942 & 19.431 & 19.886 & 25.415 & 27.258 \\
\hline \multirow{2}{*}{ CASE III } & $0.1 \times 0.1$ & 5.513 & 8.338 & 13.298 & 14.581 & 16.126 & 19.688 & 21.175 & 26.011 & 27.415 \\
& $0.2 \times 0.2$ & 5.059 & 8.176 & 12.898 & 14.300 & 16.153 & 19.491 & 20.289 & 25.921 & 26.515 \\
& $0.3 \times 0.3$ & 4.609 & 7.688 & 12.945 & 13.438 & 15.834 & 19.147 & 19.288 & 25.054 & 26.858 \\
\hline
\end{tabular}


TABLE 3: Nondimensional frequencies of the cross-ply laminate ([0/90]s) with CCCC boundary condition, $E 1 / E 2=10, a=2 \mathrm{~b}$, and $b=10 \mathrm{~h}$ and for different patch sizes and numbers.

\begin{tabular}{lcccccccccc}
\hline Modes & & 1st & 2nd & 3rd & 4th & 5th & 6th & 7th & 8th & 9th \\
\hline Present & $0 \times 0$ & 2.612 & 4.114 & 6.483 & 6.913 & 7.453 & 9.582 & 10.873 & 12.444 & 13.023 \\
\hline \multirow{2}{*}{ CASE I } & $0.1 \times 0.1$ & 2.597 & 4.114 & 6.481 & 6.882 & 7.458 & 9.580 & 10.871 & 12.392 & 13.032 \\
& $0.2 \times 0.2$ & 2.554 & 4.110 & 6.460 & 6.806 & 7.469 & 9.556 & 10.844 & 12.296 & 13.046 \\
& $0.3 \times 0.3$ & 2.495 & 4.096 & 6.384 & 6.727 & 7.475 & 9.486 & 10.754 & 12.274 & 13.024 \\
\hline \multirow{3}{*}{ CASE II } & $0.1 \times 0.1$ & 2.585 & 4.114 & 6.480 & 6.859 & 7.462 & 9.578 & 10.869 & 12.353 & 13.038 \\
& $0.2 \times 0.2$ & 2.512 & 4.107 & 6.443 & 6.732 & 7.480 & 9.537 & 10.823 & 12.189 & 13.063 \\
& $0.3 \times 0.3$ & 2.416 & 4.083 & 6.311 & 6.614 & 7.490 & 9.420 & 10.666 & 12.166 & 13.025 \\
\hline \multirow{2}{*}{ CASE III } & $0.1 \times 0.1$ & 2.566 & 4.113 & 6.478 & 6.821 & 7.467 & 9.576 & 10.867 & 12.287 & 13.048 \\
& $0.2 \times 0.2$ & 2.446 & 4.102 & 6.414 & 6.622 & 7.498 & 9.506 & 10.787 & 12.023 & 13.089 \\
& $0.3 \times 0.3$ & 2.297 & 4.061 & 6.188 & 6.463 & 7.515 & 9.320 & 10.520 & 12.019 & 13.026 \\
\hline
\end{tabular}

TABLE 4: Nondimensional frequencies of the cross-ply laminate ([0/90]s) with SSSS boundary condition, $E 1 / E 2=10$, and $a=b=10 \mathrm{~h}$ and for different patch sizes and numbers.

\begin{tabular}{lcccccccccc}
\hline Modes & & 1st & 2nd & 3rd & 4th & 5th & 6th & 7th & 8th & 9th \\
\hline Present & $0 \times 0$ & 2.519 & 4.986 & 8.515 & 9.783 & 10.077 & 13.783 & 16.700 & 18.704 & 19.911 \\
Ref. [32] & $0 \times 0$ & 2.519 & 4.986 & 8.515 & 9.783 & 10.077 & 13.783 & $/$ & 18.704 & 19.911 \\
& $0.1 \times 0.1$ & 2.500 & 4.985 & 8.513 & 9.714 & 10.088 & 13.780 & 16.685 & 18.568 & 19.906 \\
CASE I & $0.2 \times 0.2$ & 2.448 & 4.968 & 8.485 & 9.578 & 10.112 & 13.745 & 16.511 & 18.305 & 19.857 \\
& $0.3 \times 0.3$ & 2.379 & 4.908 & 8.383 & 9.507 & 10.107 & 13.671 & 16.115 & 18.193 & 19.771 \\
\hline \multirow{2}{*}{ CASE II } & $0.1 \times 0.1$ & 2.486 & 4.984 & 8.511 & 9.663 & 10.096 & 13.777 & 16.674 & 18.466 & 19.903 \\
& $0.2 \times 0.2$ & 2.399 & 4.955 & 8.462 & 9.440 & 10.136 & 13.716 & 16.366 & 18.041 & 19.820 \\
& $0.3 \times 0.3$ & 2.287 & 4.851 & 8.285 & 9.344 & 10.129 & 13.592 & 15.704 & 17.912 & 19.699 \\
\hline \multirow{2}{*}{ CASE III } & $0.1 \times 0.1$ & 2.463 & 4.982 & 8.509 & 9.581 & 10.109 & 13.773 & 16.654 & 18.297 & 19.897 \\
& $0.2 \times 0.2$ & 2.322 & 4.932 & 8.424 & 9.240 & 10.175 & 13.670 & 16.124 & 17.668 & 19.764 \\
& $0.3 \times 0.3$ & 2.155 & 4.760 & 8.127 & 9.131 & 10.163 & 13.473 & 15.089 & 17.573 & 19.615 \\
\hline
\end{tabular}

TABLE 5: Nondimensional frequencies of the cross-ply laminate ([0/90]s) with SSSS boundary condition, $E 1 / E 2=20$, and $a=b=10 \mathrm{~h}$ and for different patch sizes and numbers.

\begin{tabular}{lcccccccccc}
\hline Modes & & 1st & 2nd & 3rd & 4th & 5th & 6th & 7th & 8th & 9th \\
\hline Present & $0 \times 0$ & 2.638 & 4.917 & 9.355 & 9.637 & 10.554 & 13.826 & 16.524 & 19.668 & 20.752 \\
Ref. [32] & $0 \times 0$ & 2.638 & 4.917 & 9.354 & 9.637 & 10.554 & 13.826 & $/$ & $/$ & 20.752 \\
& $0.1 \times 0.1$ & 2.618 & 4.916 & 9.352 & 9.569 & 10.560 & 13.823 & 16.509 & 19.681 & 20.598 \\
CASE I & $0.2 \times 0.2$ & 2.564 & 4.899 & 9.321 & 9.436 & 10.573 & 13.788 & 16.338 & 19.681 & 20.286 \\
& $0.3 \times 0.3$ & 2.491 & 4.841 & 9.209 & 9.367 & 10.553 & 13.715 & 15.953 & 19.533 & 20.164 \\
\hline \multirow{2}{*}{ CASE II } & $0.1 \times 0.1$ & 2.604 & 4.915 & 9.351 & 9.519 & 10.565 & 13.820 & 16.498 & 19.691 & 20.476 \\
& $0.2 \times 0.2$ & 2.512 & 4.886 & 9.296 & 9.303 & 10.587 & 13.760 & 16.197 & 19.690 & 19.957 \\
& $0.3 \times 0.3$ & 2.395 & 4.785 & 9.101 & 9.209 & 10.553 & 13.639 & 15.557 & 19.433 & 19.828 \\
\hline \multirow{2}{*}{ CASE III } & $0.1 \times 0.1$ & 2.579 & 4.913 & 9.348 & 9.440 & 10.573 & 13.816 & 16.479 & 19.707 & 20.264 \\
& $0.2 \times 0.2$ & 2.432 & 4.864 & 9.110 & 9.254 & 10.609 & 13.714 & 15.963 & 19.481 & 19.704 \\
& $0.3 \times 0.3$ & 2.257 & 4.695 & 8.925 & 9.005 & 10.553 & 13.526 & 14.967 & 19.267 & 19.416 \\
\hline
\end{tabular}

$E 2=10$ and $a=b$. We observe that the nondimensional frequencies of SSSS plate are much smaller than those of CCCC plate. The results of plate without a patch are validated by Reddy's solution [32]. It is found that the results of present approach are exactly as same as the reference solutions. Table 5 gives the frequencies for the plate with modulus ratio $E 1 / E 2=20$. The nondimensional frequencies are a bit higher than those in Table 4 when increasing the modulus ratio. The results of plate without a patch are also well validated by Reddy's solution. The effect of aspect ratio is also investigated, and the results are given in Table 6 . When $a=2 \mathrm{~b}$, the nondimensional frequencies are apparently decreased. From these three tables, we can obtain the following conclusions. The plate with four patches, CASE III, possesses smallest lower natural frequencies compared with the other two cases, especially for the first three natural frequencies. The patch size also significantly affects the natural frequencies. It can be explained by the fact that, with surface-distributed patch, the system mass increases a lot, while the system stiffness changes slightly. This results in a 
TABLE 6: Nondimensional frequencies of the cross-ply laminate ([0/90]s) with SSSS boundary condition, $E 1 / E 2=10, a=2 \mathrm{~b}$, and $b=10 \mathrm{~h}$ and for different patch sizes and numbers.

\begin{tabular}{lcccccccccc}
\hline Modes & & 1st & 2nd & 3rd & 4th & 5th & 6th & 7th & 8th & 9th \\
\hline Present & $0 \times 0$ & 1.246 & 2.519 & 4.175 & 4.978 & 4.986 & 6.905 & 8.515 & 9.159 & 9.783 \\
& $0.1 \times 0.1$ & 1.242 & 2.519 & 4.175 & 4.959 & 4.989 & 6.904 & 8.514 & 9.126 & 9.783 \\
CASE I & $0.2 \times 0.2$ & 1.228 & 2.518 & 4.167 & 4.910 & 4.996 & 6.893 & 8.500 & 9.055 & 9.780 \\
& $0.3 \times 0.3$ & 1.208 & 2.514 & 4.140 & 4.851 & 5.004 & 6.855 & 8.448 & 9.012 & 9.772 \\
\hline \multirow{3}{*}{ CASE II } & $0.1 \times 0.1$ & 1.238 & 2.519 & 4.174 & 4.945 & 4.991 & 6.903 & 8.513 & 9.101 & 9.783 \\
& $0.2 \times 0.2$ & 1.215 & 2.517 & 4.162 & 4.862 & 5.003 & 6.884 & 8.488 & 8.979 & 9.777 \\
& $0.3 \times 0.3$ & 1.182 & 2.509 & 4.115 & 4.768 & 5.016 & 6.820 & 8.397 & 8.916 & 9.764 \\
\hline \multirow{3}{*}{ CASE III } & $0.1 \times 0.1$ & 1.232 & 2.519 & 4.174 & 4.922 & 4.994 & 6.902 & 8.512 & 9.059 & 9.782 \\
& $0.2 \times 0.2$ & 1.194 & 2.516 & 4.152 & 4.788 & 5.015 & 6.869 & 8.469 & 8.859 & 9.773 \\
& $0.3 \times 0.3$ & 1.141 & 2.502 & 4.071 & 4.650 & 5.036 & 6.762 & 8.312 & 8.780 & 9.752 \\
\hline
\end{tabular}

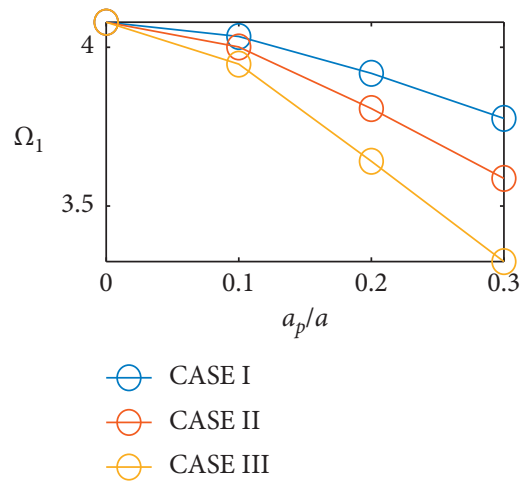

(a)

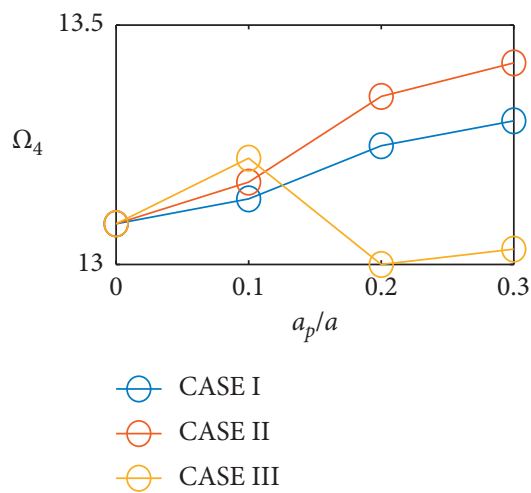

(d)

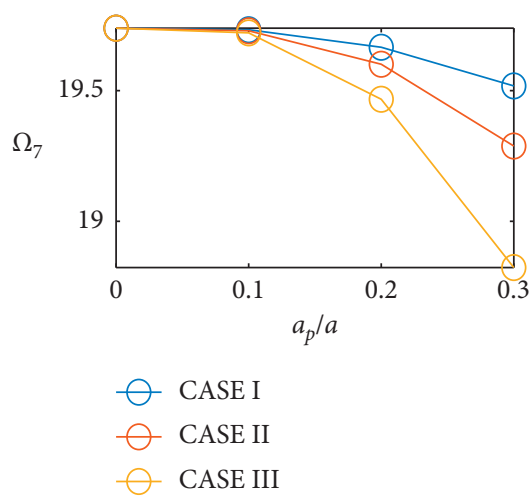

(g)

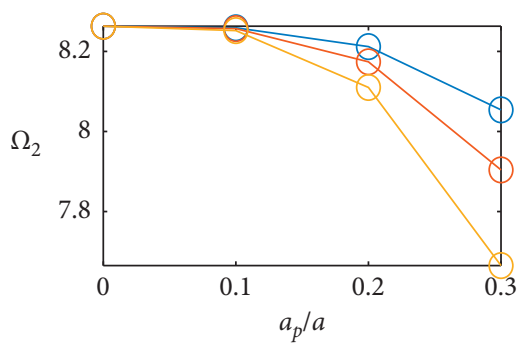

$\checkmark$ CASE I

- CASE II

- CASE III

(b)

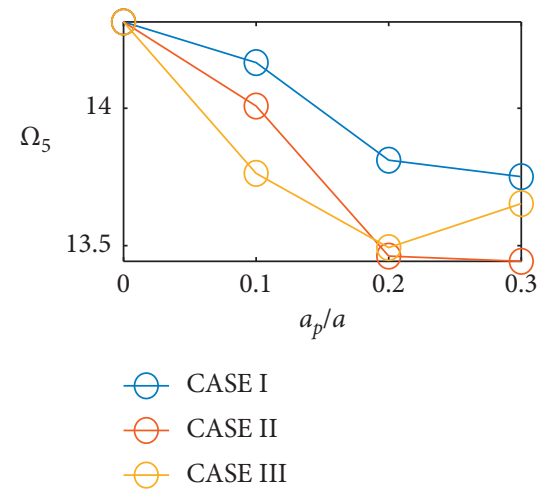

(e)

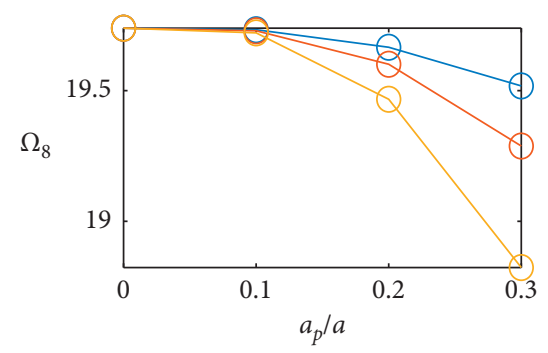

CASE I

$\checkmark$ CASE II

- CASE III

(h)

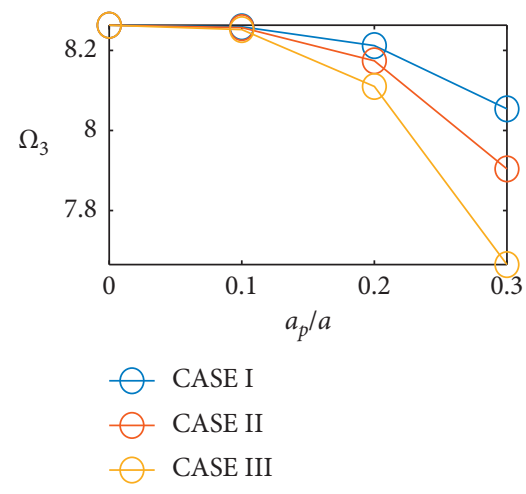

(c)

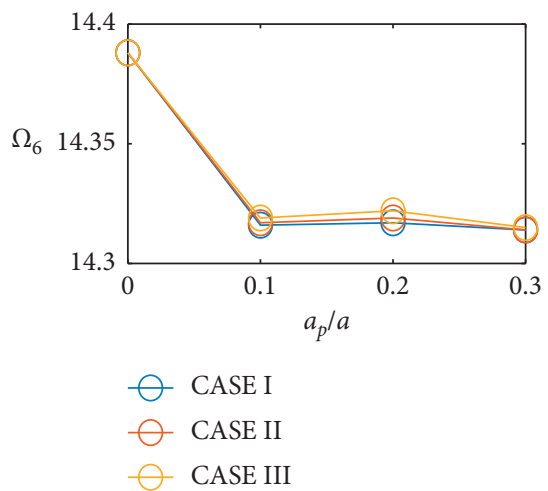

(f)

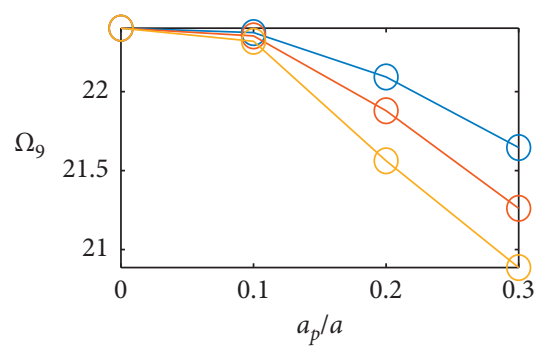

$\bigcirc$ CASE I

$\checkmark$ CASE II

- CASE III

(i)

FIgURE 4: Nondimensional frequencies of the angle-ply laminate $([45 /-45] \mathrm{s})$ with CCCC boundary condition, $E 1 / E 2=10$, and $a=b=10 \mathrm{~h}$ and for different patch sizes and numbers. 


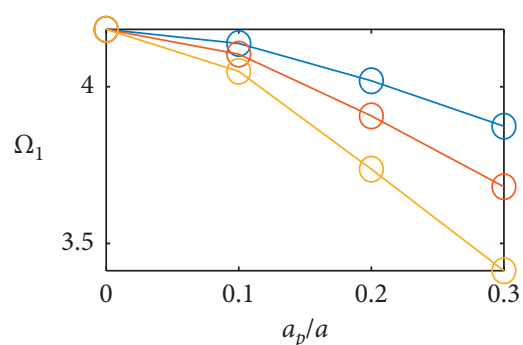

- CASE I

- CASE II

- CASE III

(a)

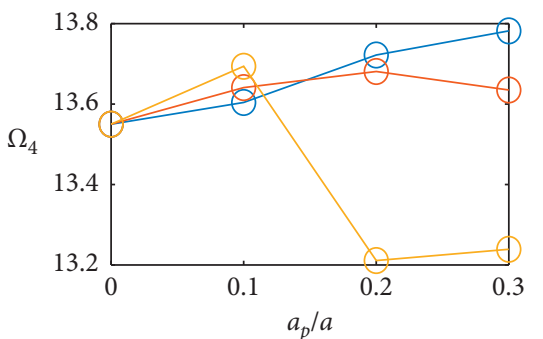

- CASE I

$\bigcirc$ CASE II

$\checkmark$ CASE III

(d)

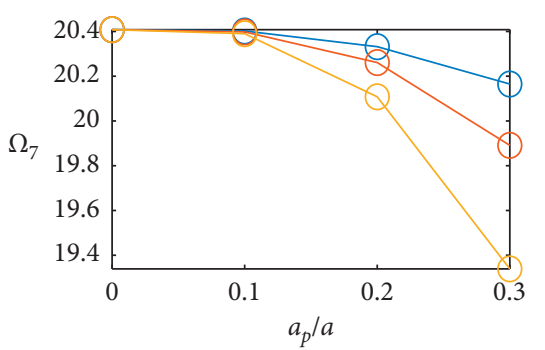

- CASE I

- CASE II

- CASE III

(g)

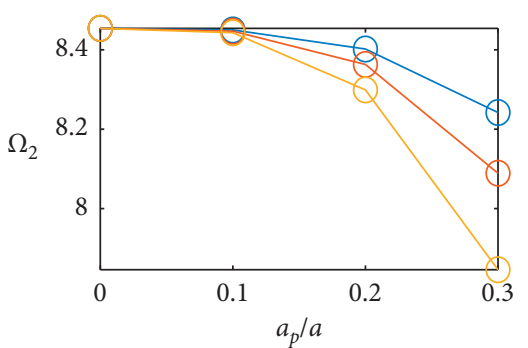

- CASE I

$\checkmark$ CASE II

CASE III

(b)

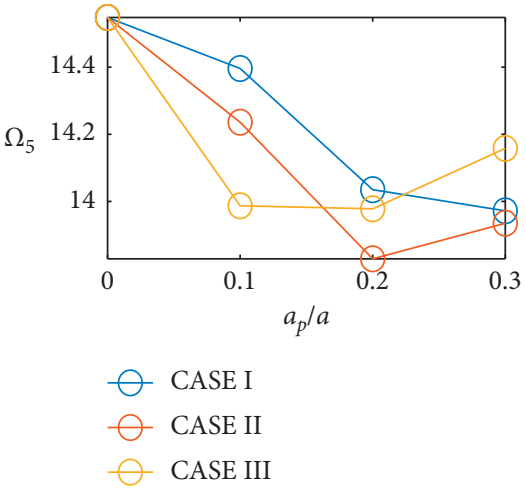

(e)

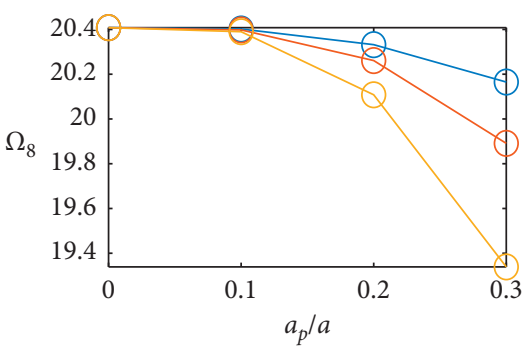

- CASE I

$\checkmark$ CASE II

$\checkmark$ CASE III

(h)

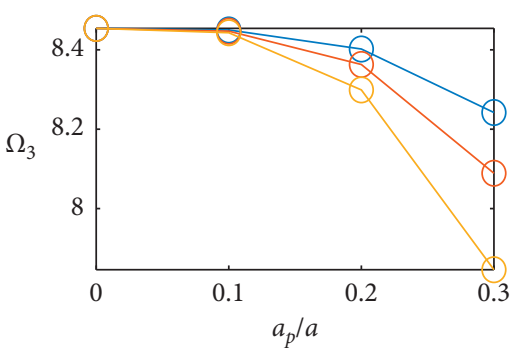

- CASE I

- CASE II

CASE III

(c)

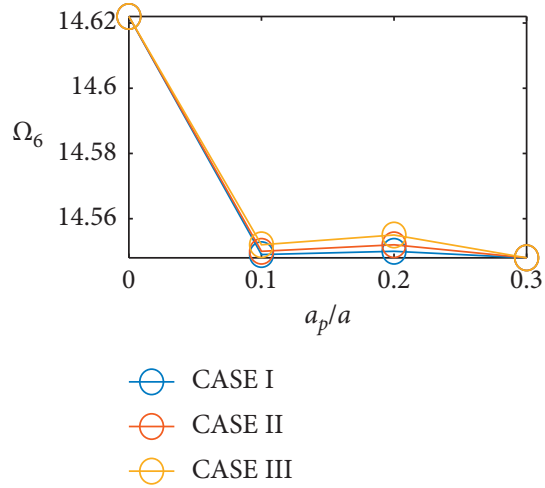

(f)

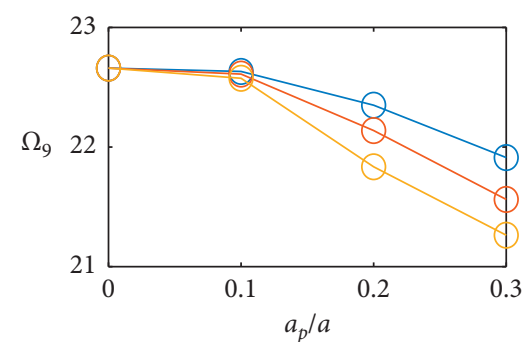

$\bigcirc$ CASE I
- CASE II
- CASE III

(i)

FIGURE 5: Nondimensional frequencies of the angle-ply laminate ([45/-45]s) with CCCC boundary condition, $E 1 / E 2=20$, and $a=b=10 \mathrm{~h}$ and for different patch sizes and numbers.

significant reduction in the lower natural frequencies. However, this effect is not clear for higher natural frequencies.

3.2. Angle-Ply Plates ([45/-45]s) with Single and Multiple Patches. In this part, we investigate the natural frequencies of angle-ply plates ([45/-45]s) with single and multiple patches by using the graphical descriptions. Angle-ply laminates are also very important in engineering structures since they can sustain large shear forces. We firstly examine the four-edge clamped
(CCCC) plate with different patch numbers and sizes, as shown in Figures 4-6. We also present the first nine nondimensional frequencies for each case, and they are drawn together to make comparison. From these three figures, it is found that the natural frequencies are smaller than those of cross-ply cases. The main tendency of the nondimensional frequency is that it decreases with the increase in patch size, except for $\Omega_{4}$ in Figures 4 and 5 and $\Omega_{6}$ and $\Omega_{9}$ in Figure 6. In general, most of the frequencies of CASE I are larger than those of CASE II and CASE III and the relative difference is very significant when the patch size $a_{d} / a$ equals 0.2 or 0.3 . The modulus and aspect ratio 


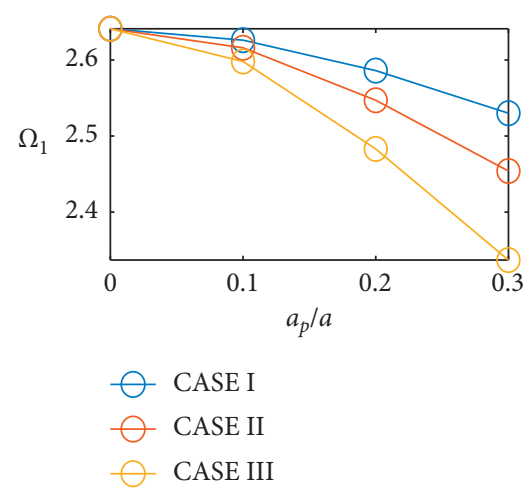

(a)

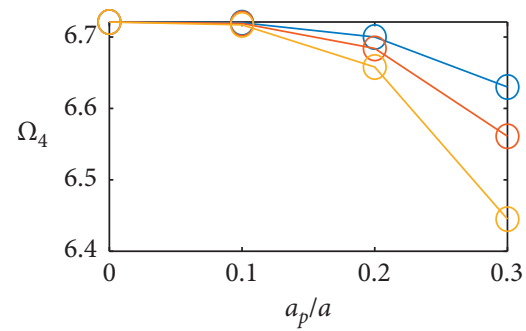

O CASE I

$\checkmark$ CASE II

$\checkmark$ CASE III

(d)

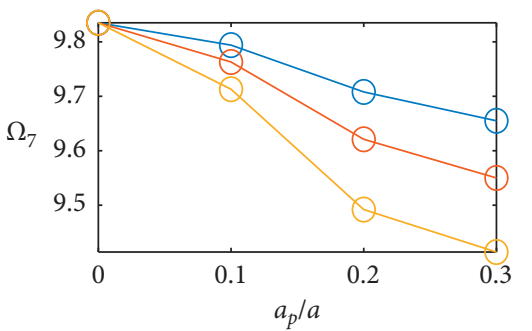

- CASE I

- CASE II

- CASE III

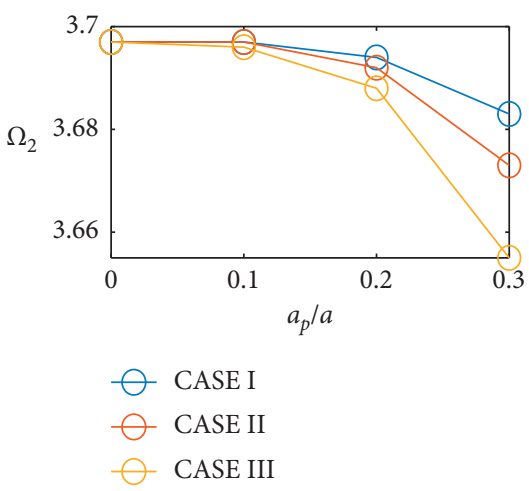

(b)

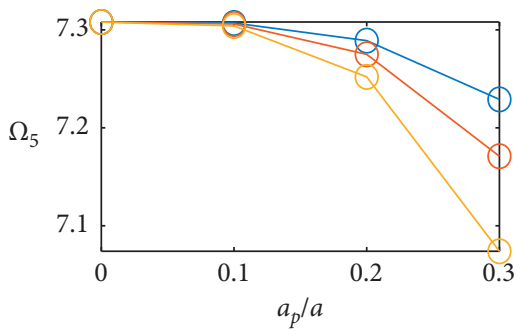

$\bigcirc$ CASE I

$\checkmark$ CASE II

$\checkmark$ CASE III

(e)

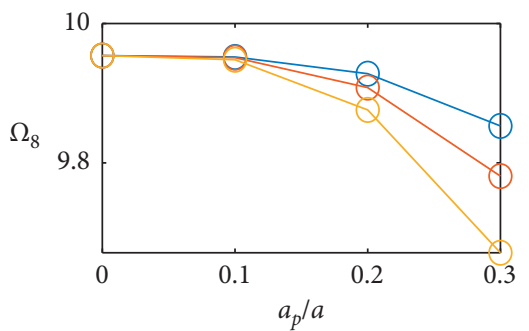

- CASE I

$\checkmark$ CASE II

$\checkmark$ CASE III

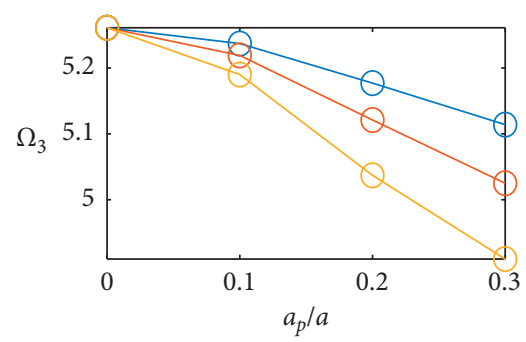

- CASE I

$\checkmark$ CASE II

$\checkmark$ CASE III

(c)

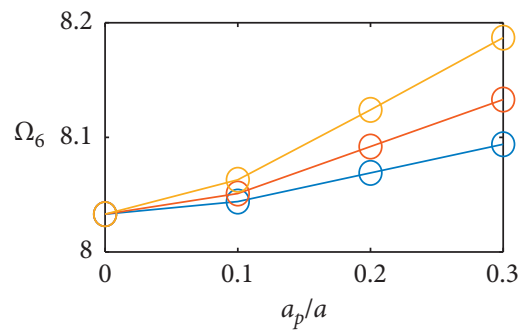

- CASE I

$\checkmark$ CASE II

- CASE III

(f)

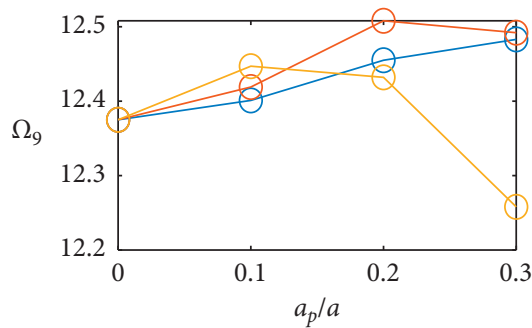

$\bigcirc$ CASE I

$\checkmark$ CASE II

- CASE III

(i)

Figure 6: Nondimensional frequencies of the angle-ply laminate ([45/-45]s) with CCCC boundary condition, $E 1 / E 2=10, a=2 \mathrm{~b}$, and $b=10 \mathrm{~h}$ and for different patch sizes and numbers. 


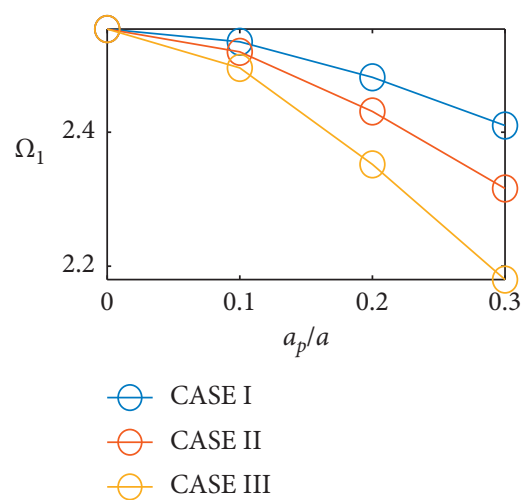

(a)

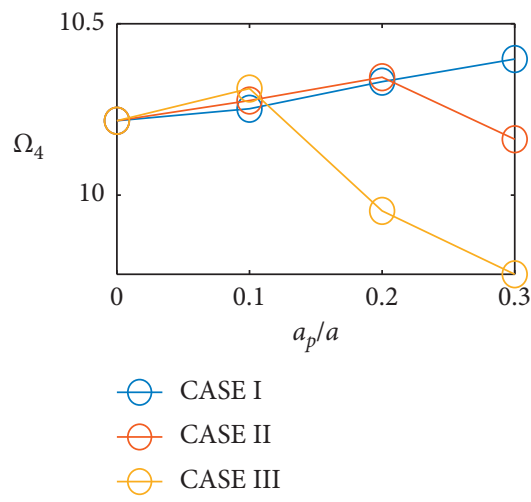

(d)

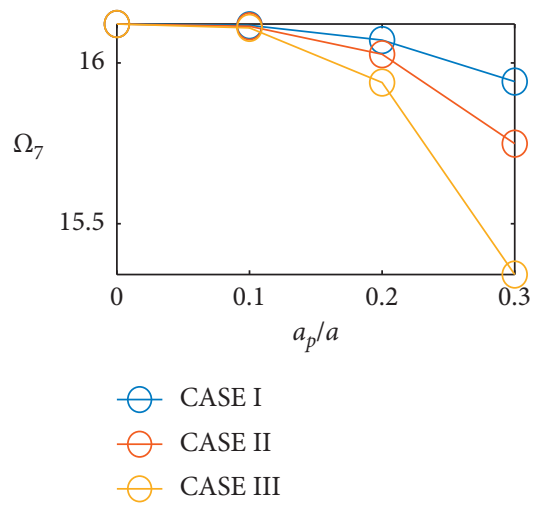

(g)

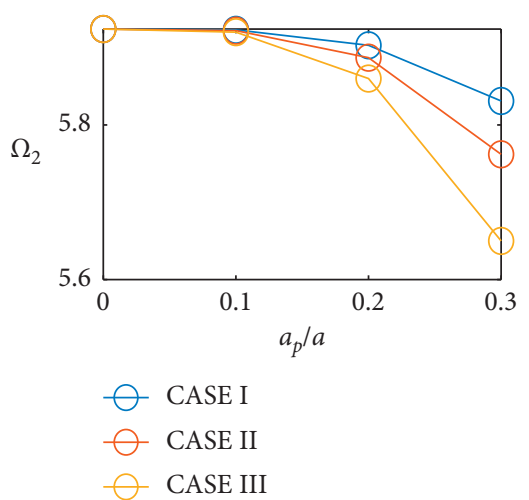

(b)

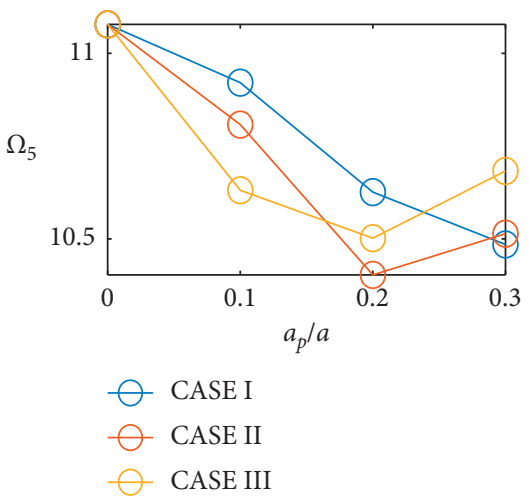

(e)

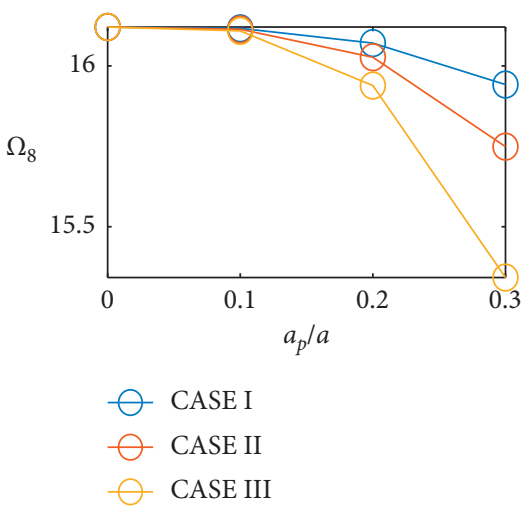

(h)

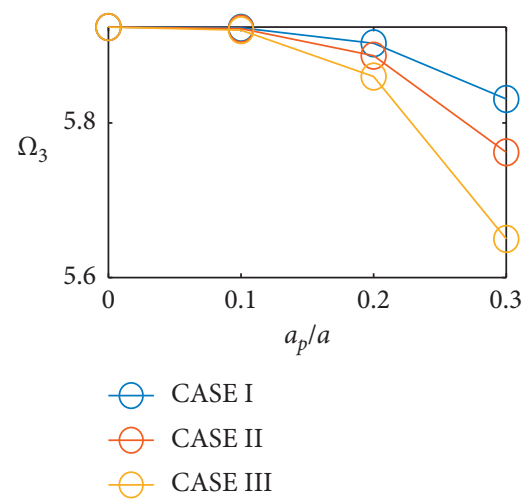

(c)

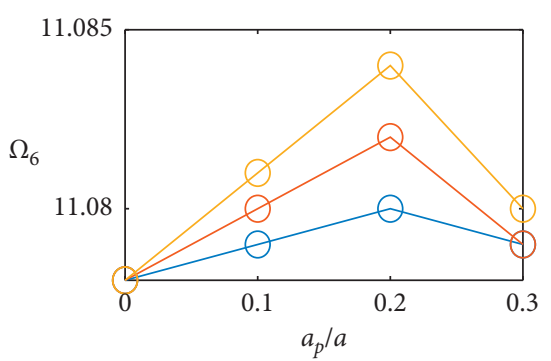

$\bigcirc$ CASE I

- CASE II

O CASE III

(f)

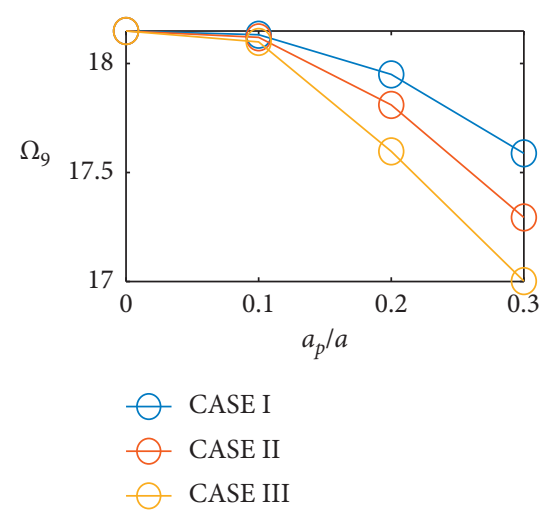

(i)

FIGURE 7: Nondimensional frequencies of the angle-ply laminate ([45/-45]s) with SSSS boundary condition, $E 1 / E 2=10$, and $a=b=10 \mathrm{~h}$ and for different patch sizes and numbers.

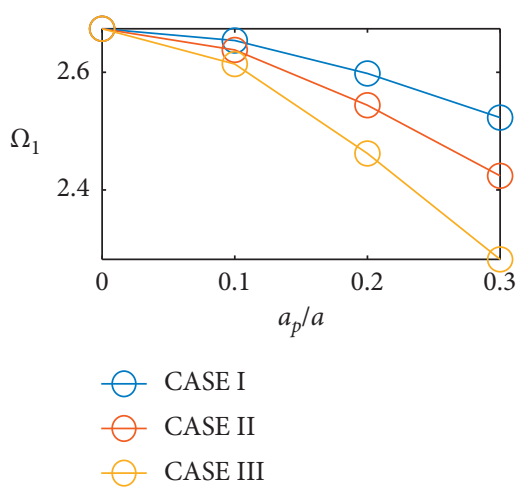

(a)
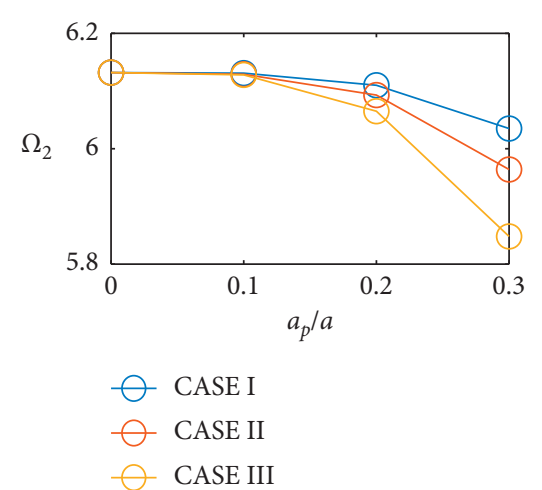

(b)

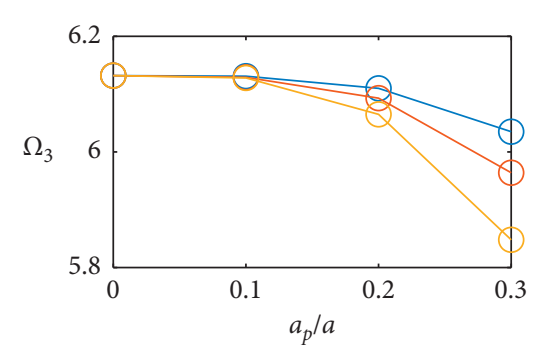

- CASE I

CASE II

- CASE III

(c)

Figure 8: Continued. 

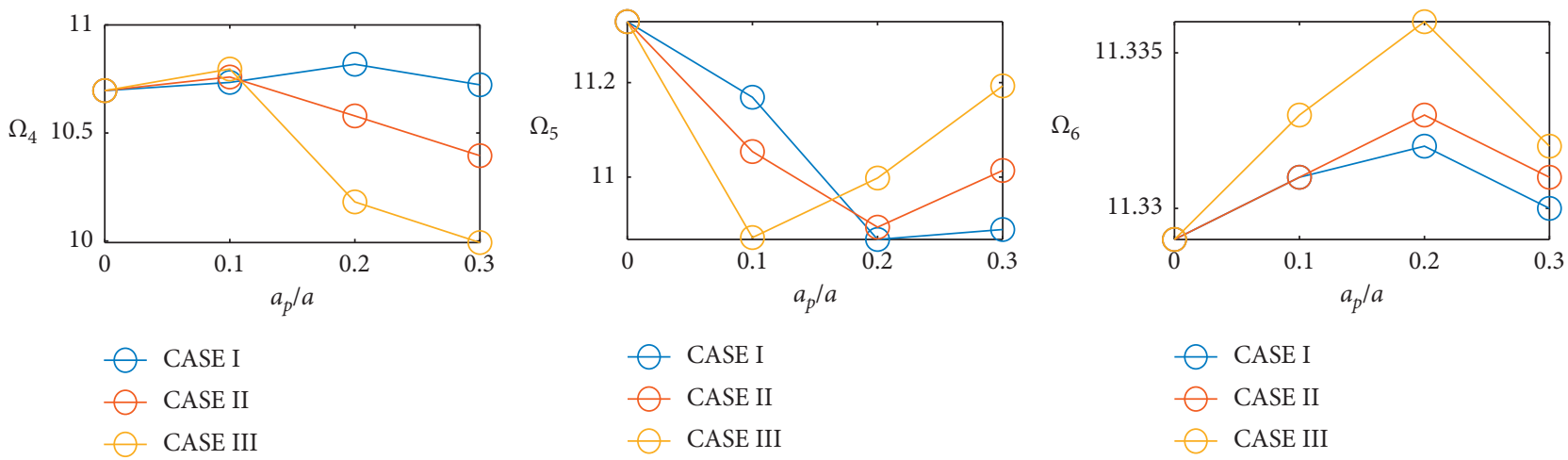

(d)

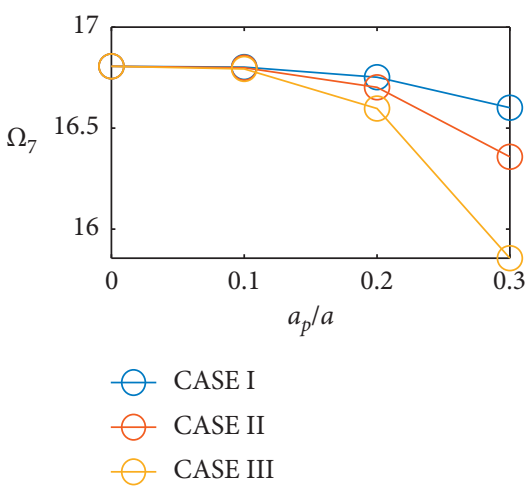

(g)

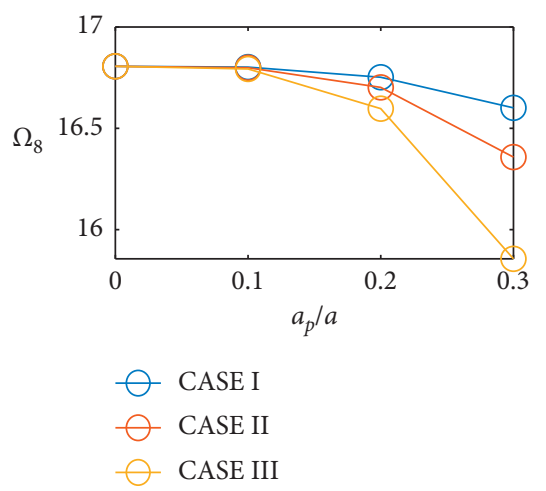

(h)

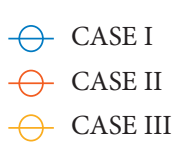

(f)

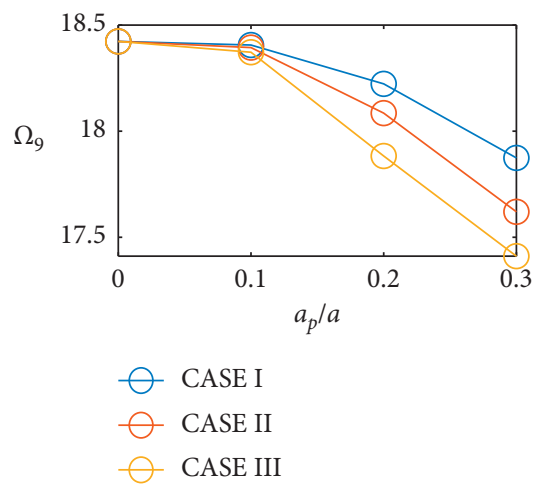

(i)

FIGURE 8: Nondimensional frequencies of the angle-ply laminate ([45/-45]s) with SSSS boundary condition, $E 1 / E 2=20$, and $a=b=10 \mathrm{~h}$ and for different patch sizes and numbers.

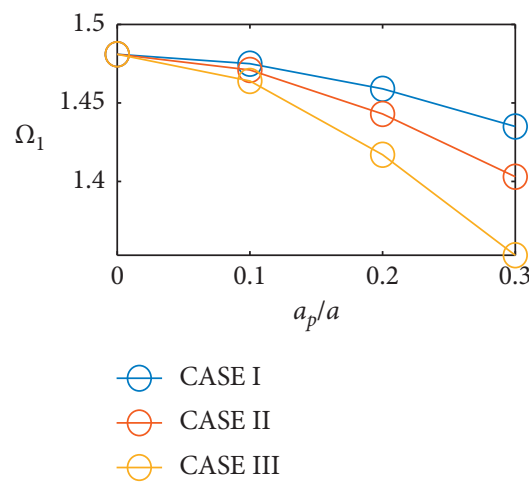

(a)

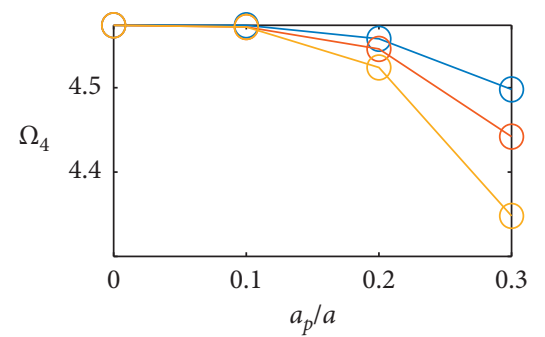

- CASE I

- CASE II

CASE III

(d)

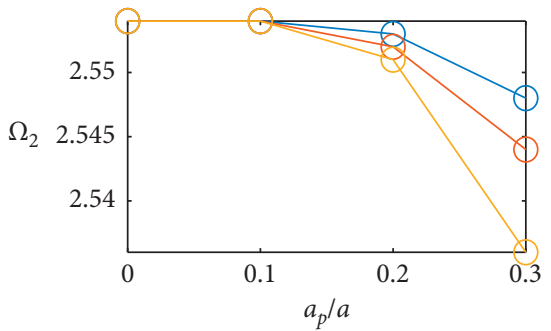

$\bigcirc$ CASE I

$\bigcirc$ CASE II

- CASE III

(b)

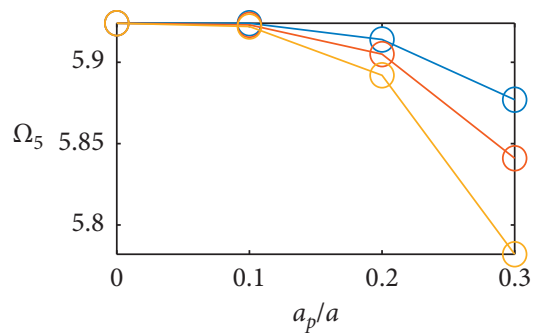

- CASE I

$\checkmark$ CASE II

CASE III

(e)

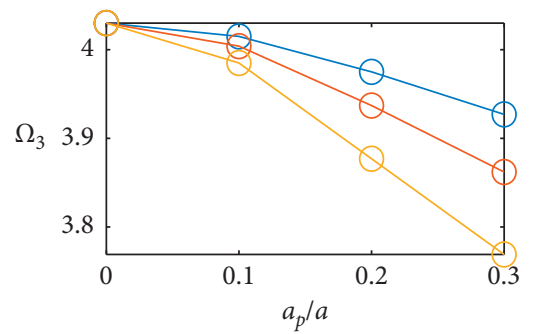

$\bigcirc$ CASE I

$\checkmark$ CASE II

- CASE III

(c)

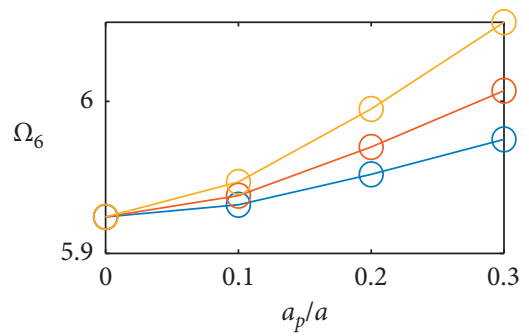

$\bigcirc$ CASE I

$\checkmark$ CASE II

$\checkmark$ CASE III

(f)

Figure 9: Continued. 


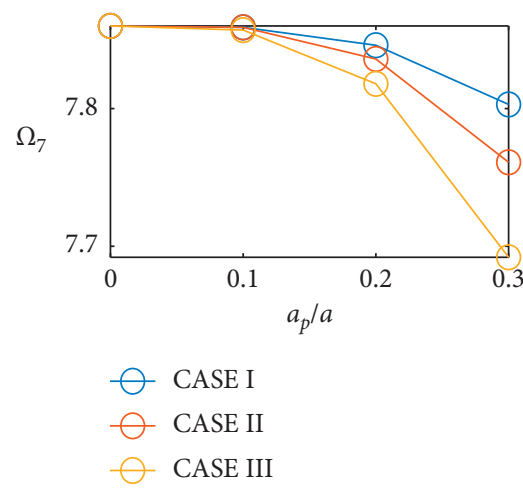

(g)

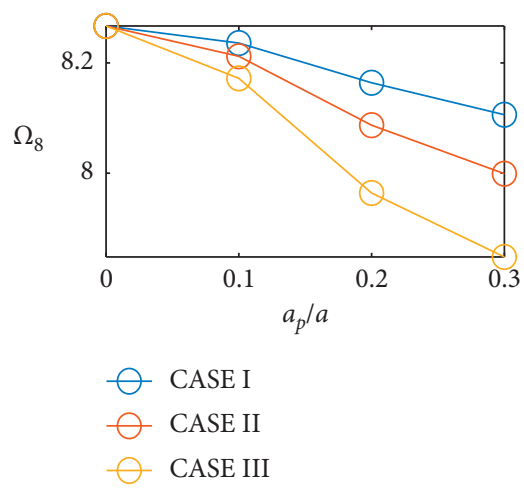

(h)

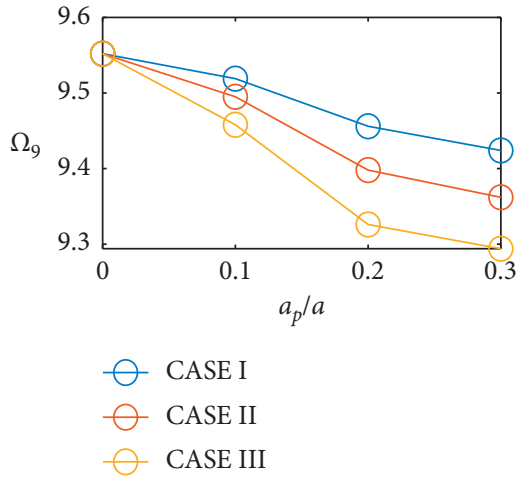

(i)

FIGURE 9: Nondimensional frequencies of the angle-ply laminate ([45/-45]s) with SSSS boundary condition, $E 1 / E 2=10, a=2 \mathrm{~b}$, and $b=10 \mathrm{~h}$ and for different patch sizes and numbers.

also have effects on the nondimensional frequencies as discussed before.

Finally, the results of four-edge simply supported (SSSS) angle-ply plate with single and multiple patches are also investigated, and the results are shown in Figures 7-9. For this example, we observe that CASE I also possesses larger frequencies than CASE II and CASE III. However, for $\Omega_{6}$, the conclusion is opposite where CASE III possesses largest values, while CASE I possesses smallest values. The results show that, with more patches and larger patch size on the substrate angleply laminate, the natural frequencies decrease as a consequence. Readers need to be reminded that all conclusions obtained in this work are based on the patches located at the center or symmetrically located on the plate. For arbitrarily located patches, we need to use the present approach for further investigations.

\section{Conclusions}

In this paper, we investigated the transverse vibration problem of composite plates with surface-distributed multiple composite patches by means of the Rayleigh-Ritz method and independent coordinate systems. Numerical solutions of the nondimensionalized natural frequencies of rectangular plates with various layer stacking sequences, modulus ratios, aspect ratios, and boundary conditions are calculated and compared with the existed benchmark solutions and FEM solutions. The equations of motion are obtained from Lagrange's equation in which the total energies are calculated by the summation of the energies of substrate plate and the energies of composite patches in this work. It is necessary to build the connections between different coordinate systems by applying the displacement-matching condition. The benefit of using independent coordinate systems is that it is more convenient to build the mathematical modeling. The convergent results are obtained by using only 10 admissible functions in both $x$ and $y$ directions. For the FEM validation, it often requires to use very fine mesh to obtain convergent frequency results, while it is still hard to acquire accurate high frequencies and vibration modes. Computationally, it is very convenient and efficient to calculate the natural frequencies of laminated plates with multiple patches by using the present method. The numerical results are well validated and demonstrate the feasibility of using the present approach to solve the vibration problem of composite plates with multiple patches.

\section{Data Availability}

The data used to support the findings of this study are available from the corresponding author upon request.

\section{Conflicts of Interest}

The authors declare that they have no conflicts of interest.

\section{Acknowledgments}

This work was supported by the National Natural Science Foundation of China (Grant nos. 11702150 and 11672142), Natural Science Foundation of Zhejiang Province (Grant no. LY21A020003), Natural Science Foundation of Ningbo (Grant nos. 202003N4015 and 202003N4163), the project of Key Laboratory of Impact and Safety Engineering (Ningbo University), Ministry of Education, China (Grant no. CJ202009), the Technology Innovation 2025 Program of Municipality of Ningbo (Grant no. 2019B10122), the research fund by the College of Science \& Technology, Ningbo University (Grant no. 003/yk202018), and the K. C. Wong Magna Fund through Ningbo University.

\section{References}

[1] R. Jones, A. Baker, N. Matthews, and V. Champagne, Aircraft Sustainment and Repair, Butterworth-Heinemann, Oxford, UK, 2018.

[2] V. M. Karbhari, Rehabilitation of Metallic Civil Infrastructure Using Fiber Reinforced Polymer (FRP) Composites, Woodhead Publishing, Cambridge, England, 2014.

[3] M. A. Obohat, E. Tahvilian, M. E. Yildizdag, and A. Ergin, "Three-dimensional multi-patch isogeometric analysis of composite laminates with a discontinuous Galerkin approach," Journal of Engineering for the Maritime Environment, Article ID 1475090220925734, 2020. 
[4] Y. Niu, X. Li, Z. Mu, and T. Liu, "Stress analysis of aluminium plates one-sided adhesively bonded reinforced with square composite patches using state space method," Journal of Adhesion Science and Technology, vol. 30, no. 22, pp. 24852497, 2016.

[5] W.-C. Wang and J.-S. Hsu, "Investigation of the size effect of composite patching repaired on edge-cracked plates," Composite Structures, vol. 49, no. 4, pp. 415-423, 2000.

[6] Y. Zhu, J. Liu, D. Liu et al., "Fiber path optimization based on a family of curves in composite laminate with a center hole," Composites Part B: Engineering, vol. 111, pp. 91-102, 2017.

[7] I. Ivañez, S. K. Garcia-Castillo, S. Sanchez-Saez, and E. Barbero, "Experimental study of the impact behavior of repaired thin laminates with double composite patch," $\mathrm{Me}$ chanics of Advanced Materials and Structures, vol. 27, no. 19, pp. 1701-1708, 2020.

[8] J.-D. Mathias and M. Grédiac, "Applying the grid method to validate a numerical model predicting the transverse shear stress distributions in patched structures," Composites Part A: Applied Science and Manufacturing, vol. 39, no. 8, pp. 11941209, 2008.

[9] A. Baker, "Bonded composite repair of fatigue-cracked primary aircraft structure," Composite Structures, vol. 47, no. 1-4, pp. 431-443, 1999.

[10] J. J. Schubbe and S. Mall, "Investigation of a cracked thick aluminum panel repaired with a bonded composite patch," Engineering Fracture Mechanics, vol. 63, no. 3, pp. 305-323, 1999.

[11] J. Cho and C. T. Sun, "Lowering thermal residual stresses in composite patch repairs in metallic aircraft structure," AIAA Journal, vol. 39, no. 10, pp. 2013-2018, 2001.

[12] J. Lee, M. Cho, and H. S. Kim, "Bending analysis of a laminated composite patch considering the free-edge effect using a stress-based equivalent single-layer composite model," International Journal of Mechanical Sciences, vol. 53, no. 8, pp. 606-616, 2011.

[13] H. S. Kim, M. Cho, J. Lee, A. Deheeger, M. Grédiac, and J.-D. Mathias, "Three dimensional stress analysis of a composite patch using stress functions," International Journal of Mechanical Sciences, vol. 52, no. 12, pp. 1646-1659, 2010.

[14] W.-C. Wang and C.-H. Chen, "Investigation of vibration behavior of patched edge-cracked composite plates," Journal of Reinforced Plastics and Composites, vol. 21, no. 6, pp. 533-557, 2002.

[15] I. S. Sadek, J. C. Bruch, J. M. Sloss, and S. Adali, "Feedback control of vibrating plates using piezoelectric patch sensors and actuators," Composite Structures, vol. 62, no. 3-4, pp. 397-402, 2003.

[16] T. Roy and D. Chakraborty, "Genetic algorithm based optimal control of smart composite shell structures under mechanical loading and thermal gradient," Smart Materials and Structures, vol. 18, no. 11, p. 115006, 2009.

[17] A. Paknejad, G. Rahimi, A. Farrokhabadi, and M. M. Khatibi, "Analytical solution of piezoelectric energy harvester patch for various thin multilayer composite beams," Composite Structures, vol. 154, pp. 694-706, 2016.

[18] P. Tan and L. Tong, "Multiple delamination detection of a composite beam using magnetostrictive patch," AIAA Journal, vol. 44, no. 11, pp. 2547-2551, 2006.

[19] B. Huang, H. S. Kim, and G. H. Yoon, "Modeling of a partially debonded piezoelectric actuator in smart composite laminates," Smart Materials and Structures, vol. 24, no. 7, Article ID 075013, 2015.
[20] B. Huang, H. S. Kim, and B. D. Youn, "Active vibration control of smart composite laminates with partial debonding of actuator," International Journal of Precision Engineering and Manufacturing, vol. 16, no. 4, pp. 831-840, 2015.

[21] B. Huang, H. S. Kim, and G. H. Yoon, "Investigation of actuator debonding effects on active control in smart composite laminates," Advances in Mechanical Engineering, vol. 7, no. 4, Article ID 1687814015578363, 2015.

[22] A. L. Araújo, V. S. Carvalho, C. M. Mota Soares, J. Belinha, and A. J. M. Ferreira, "Vibration analysis of laminated soft core sandwich plates with piezoelectric sensors and actuators," Composite Structures, vol. 151, pp. 91-98, 2016.

[23] S. L. Schulz, H. M. Gomes, and A. M. Awruch, "Optimal discrete piezoelectric patch allocation on composite structures for vibration control based on GA and modal LQR," Computers \& Structures, vol. 128, pp. 101-115, 2013.

[24] A. Alibeigloo, M. Shakeri, and M. R. Kari, "Free vibration analysis of antisymmetric laminated rectangular plates with distributed patch mass using third-order shear deformation theory," Ocean Engineering, vol. 35, no. 2, pp. 183-190, 2008.

[25] A. Alibeigloo and M. R. Kari, "Forced vibration analysis of antisymmetric laminated rectangular plates with distributed patch mass using third order shear deformation theory," ThinWalled Structures, vol. 47, no. 6-7, pp. 653-660, 2009.

[26] A. Vaziri and H. Nayeb-Hashemi, "Dynamic response of a repaired composite beam with an adhesively bonded patch under a harmonic peeling load," International Journal of Adhesion and Adhesives, vol. 26, no. 5, pp. 314-324, 2006.

[27] B. Safaei, R. Moradi-Dastjerdi, K. Behdinan, and F. Chu, "Critical buckling temperature and force in porous sandwich plates with CNT-reinforced nanocomposite layers," Aerospace Science and Technology, vol. 91, pp. 175-185, 2019.

[28] Y. Kiani, "Free vibration of FG-CNT reinforced composite skew plates," Aerospace Science and Technology, vol. 58, pp. 178-188, 2016.

[29] B. Huang, J. Wang, J. Du, T. Ma, Y. Guo, and Z. Qian, "Vibration analysis of a specially orthotropic composite laminate with rectangular cutout using independent coordinate coupling method," Composite Structures, vol. 150, pp. 53-61, 2016.

[30] Y. Guo, Y. Jiang, and B. Huang, "Independent coordinate coupling method for vibration analysis of a functionally graded carbon nanotube-reinforced plate with central hole," Advances in Mechanical Engineering, vol. 11, no. 8, Article ID $1687814019872924,2019$.

[31] M. Cho and H. S. Kim, "Iterative free-edge stress analysis of composite laminates under extension, bending, twisting and thermal loadings," International Journal of Solids and Structures, vol. 37, no. 3, pp. 435-459, 2000.

[32] J. N. Reddy, Mechanics of Laminated Composite Plates and Shells: Theory and Analysis, CRC Press, Boca Raton, FL, USA, 2nd edition, 2004. 